\title{
Lensing Reconstruction with CMB Temperature and Polarization
}

\author{
Michael Kesden, Asantha Cooray, and Marc Kamionkowski \\ Theoretical Astrophysics, California Institute of Technology, Pasadena, California 91125
}

\begin{abstract}
Weak gravitational lensing by intervening large-scale structure induces a distinct signature in the cosmic microwave background (CMB) that can be used to reconstruct the weak-lensing displacement map. Estimators for individual Fourier modes of this map can be combined to produce an estimator for the lensing-potenial power spectrum. The naive estimator for this quantity will be biased upwards by the uncertainty associated with reconstructing individual modes; we present an iterative scheme for removing this bias. The variance and covariance of the lensing-potenial power spectrum estimator are calculated and evaluated numerically in a $\Lambda \mathrm{CDM}$ universe for Planck and future polarizationsensitive CMB experiments.
\end{abstract}

\section{INTRODUCTION}

The primordial cosmic microwave background (CMB) was generated when photons first decoupled from the baryonic fluid when the universe was only 400,000 years old. The vast majority of these photons travel unperturbed to the present day, and features of their angular power spectrum such as acoustic peaks and the damping tail [1] record valuable information about cosmological parameters [2]. Baryons and dark matter evolve from small inhomogeneities at decoupling into increasingly complicated large-scale structure which can subtly perturb the observed pattern of CMB anisotropies. Assuming that the primordial CMB is Gaussian, non-Gaussian correlations in the observed map can be used to reconstruct the intervening large-scale structure [3]. In addition to the importance of learning about the large-scale structure itself, reconstruction of the weak-lensing potential generated by structure is essential to constraining tensor perturbations. Weak lensing converts a fraction of the E-mode polarization generated by scalar perturbations at the last-scattering surface into B-mode polarization in the observed map. Only by subtracting this B-mode polarization can one conclusively detect the primordial B-modes which serve as a model-independent signal of tensor perturbations [4]. Understanding lensing reconstruction requires a more detailed discussion of how weak lensing affects the CMB.

Weak gravitational lensing deflects the paths of CMB photons as they travel from the last-scattering surface to the observer. This deflection is accomplished by a projected lensing potential which is a weighted line-of-sight integral of the gravitational potential between the observer and the surface of last-scattering. At each point on the sky, lensing remaps the temperature and polarization to that of a nearby point at the last-scattering surface, the deflection angle being the gradient of the aforementioned projected lensing potential. Assuming that this deflection angle is small, the temperature at any point can be expanded in a Taylor series in the gradient of the lensing potential. In Fourier space, this expansion appears as a series of convolutions of individual temperature and projected potential modes. The observed temperature-squared map in Fourier space also appears as a convolution of individual Fourier modes. Subject to an overall normalization dependent on the scale of the Fourier mode, these convolutions cancel in such a manner that each Fourier mode of the temperature-squared map acts as an estimator for the same Fourier mode of the projected lensing potential.

Lensing reconstruction as outlined above has been considered previously $[5,6]$. In these works, two sources of noise were identified, and a filter of the temperature-squared map in Fourier space was chosen to minimize the variance associated with lensing reconstruction subject to these noise sources. The first source is intrinsic signal variance; the observed large-scale structure is one arbitrary member of an ensemble of realizations allowed by theory. The second source of noise, endemic to this method of lensing reconstruction, is a consequence of the nature of the primordial CMB. Like the large-scale structure itself, the pattern of CMB anisotropies at the last-scattering surface is only one of many possible realizations allowed by theory. We do not know a priori which of these realizations nature has provided us, and this uncertainty hinders our ability to deconvolve the effects of lensing from true anisotropies at the last-scattering surface. Even if the true pattern of anisotropies at the last-scattering surface was known, the finite amount of power in the CMB at small scales would still constrain lensing reconstruction. Silk damping at the last-scattering surface suppresses CMB power at small scales, while the finite resolution of any real experiment would limit the detection of any signal that is present at small scales. Lensing reconstruction fails below scales at which there is sufficient power, for the same reason that any remapping is indistinguishable given a uniform background.

Here, we consider a third source of noise neglected in previous studies. The filtered temperature-squared map is an unbiased estimator for the lensing potential in the approximation that a correlation between two given temperature modes is induced only by the single lensing mode whose wavevector is the sum of that of the two temperature modes. In actuality, any combination of two or more lensing modes whose wavevectors sum to this total induce correlations 
between the two temperature modes. There are many such combinations, but since these correlations add incoherently we do not expect a systematic bias. Nonetheless, for estimators of each individual lensing mode we must use our knowledge of other lensing modes to subtract off this unwanted bias. This is an iterative process, and since our knowledge of the lensing map is imperfect it induces noise in lensing reconstruction. We calculate this additional variance for various estimators constructed from CMB temperature and polarization maps, and show how it compares to the dominant noise sources for an all-sky CMB experiment with a noise-equivalent temperature of $1 \mu \mathrm{K} \sqrt{\mathrm{sec}}$. Since the lensing-potential power spectrum is a measure of the theoretical uncertainty with which we can predict the value of a given lensing mode, this noise associated with lensing reconstruction causes a systematic overestimation of the lensing-potential power spectrum. This systematic bias must be accounted for in order to compare observations with theoretical predictions.

This paper is organized as follows. In $\S \mathrm{II}$, we define the formalism we will use to explore the effects of weak lensing on the CMB. The Taylor expansion of the lensed CMB map in gradients of the lensing potential is given in both real and Fourier space, and the power spectra and trispectra of various components of the CMB temperature map are listed for later use. In $\S$ III, we show that the Fourier modes of the temperature-squared map when properly filtered can serve as estimators for the Fourier modes of the displacement map with the same wave vector. Using the power spectrum and trispectrum given in the preceding Section, we calculate the variance associated with this estimator including a new component neglected in previous studies. This variance is evaluated numerically using the currently favored $\Lambda$ CDM cosmological model with baryon density $\Omega_{b}=0.05$, matter density $\Omega_{m}=0.35$, cosmological constant density $\Omega_{\Lambda}=0.65$, Hubble parameter $h=0.65$, and power-spectrum amplitude $\sigma_{8}=0.9$. We then use the displacement estimator for individual Fourier modes to construct an unbiased estimator for the lensing-potential power spectrum in $\S \mathrm{IV}$, and calculate the variance and covariance associated with this estimator. A few concluding remarks about the implications of our work for future studies are given in $\S \mathrm{V}$. The Appendix contains useful formulae related to additional estimators of lensing based on polarization and a combination of temperature and polarization.

\section{WEAK LENSING OF THE CMB}

We consider weak lensing under the flat-sky approximation following Refs. [7,8]. As discussed before [8,9], weak lensing deflects the path of $\mathrm{CMB}$ photons resulting in a remapping of the observed temperature pattern on the sky,

$$
\begin{aligned}
\tilde{\Theta}(\hat{\mathbf{n}}) & =\Theta[\hat{\mathbf{n}}+\nabla \phi(\hat{\mathbf{n}})] \\
& \approx \Theta(\hat{\mathbf{n}})+\nabla_{i} \phi(\hat{\mathbf{n}}) \nabla^{i} \Theta(\hat{\mathbf{n}})+\frac{1}{2} \nabla_{i} \phi(\hat{\mathbf{n}}) \nabla_{j} \phi(\hat{\mathbf{n}}) \nabla^{i} \nabla^{j} \Theta(\hat{\mathbf{n}})+\ldots
\end{aligned}
$$

where $\Theta(\hat{\mathbf{n}})$ is the unlensed primary component of the CMB in a direction $\hat{\mathbf{n}}$ at the last scattering surface. The observed, gravitationally-lensed temperature map $\tilde{\Theta}(\hat{\mathbf{n}})$ in direction $\hat{\mathbf{n}}$ is that of the unlensed map in direction $\hat{\mathbf{n}}+$ $\nabla \phi(\hat{\mathbf{n}})$ where $\nabla \phi(\hat{\mathbf{n}})$ represents the lensing deflection angle or displacement map. Although a real CMB map will include secondary contributions such as the SZ effect [10], we assume that such effects can be distinguished by their frequency dependence [11]. They will not be further considered in this paper. A noise component denoted by $\Theta^{\mathrm{n}}(\hat{\mathbf{n}})$ due to finite experimental sensitivity must be included as well. Thus the total observed CMB anisotropy will be $\Theta^{\mathrm{t}}(\hat{\mathbf{n}})=\tilde{\Theta}(\hat{\mathbf{n}})+\Theta^{\mathrm{n}}(\hat{\mathbf{n}})$.

Taking the Fourier transform of the lensed map $\tilde{\Theta}(\hat{\mathbf{n}})$ under the flat-sky approximation, we write

$$
\begin{aligned}
\tilde{\Theta}(\mathbf{l}) & =\int d \hat{\mathbf{n}} \tilde{\Theta}(\hat{\mathbf{n}}) e^{-i \mathbf{l} \cdot \hat{\mathbf{n}}} \\
& =\Theta(\mathbf{l})-\int \frac{d^{2} \mathbf{l}^{\prime}}{(2 \pi)^{2}} \Theta\left(\mathbf{l}^{\prime}\right) L\left(\mathbf{l}, \mathbf{l}^{\prime}\right),
\end{aligned}
$$

where

$$
\begin{aligned}
L\left(\mathbf{l}, \mathbf{l}^{\prime}\right) \equiv \phi & \left(\mathbf{l}-\mathbf{l}^{\prime}\right)\left[\left(\mathbf{l}-\mathbf{l}^{\prime}\right) \cdot \mathbf{l}^{\prime}\right]+\frac{1}{2} \int \frac{d^{2} \mathbf{l}^{\prime \prime}}{(2 \pi)^{2}} \phi\left(\mathbf{l}^{\prime \prime}\right) \\
& \times \phi\left(\mathbf{l}-\mathbf{l}^{\prime}-\mathbf{l}^{\prime \prime}\right)\left(\mathbf{l}^{\prime \prime} \cdot \mathbf{l}^{\prime}\right)\left[\left(\mathbf{l}^{\prime \prime}+\mathbf{l}^{\prime}-\mathbf{l}\right) \cdot \mathbf{l}^{\prime}\right]+\ldots .
\end{aligned}
$$

CMB correlations in Fourier space can be described in terms of a power spectrum and trispectrum as defined in the usual manner, 


$$
\begin{aligned}
\left\langle\Theta^{i}\left(\mathbf{l}_{1}\right) \Theta^{i}\left(\mathbf{l}_{2}\right)\right\rangle & \equiv(2 \pi)^{2} \delta_{\mathrm{D}}\left(\mathbf{l}_{1}+\mathbf{l}_{2}\right) C_{l}^{i}, \\
\left\langle\Theta^{i}\left(\mathbf{l}_{1}\right) \ldots \Theta^{i}\left(\mathbf{l}_{4}\right)\right\rangle_{c} & \equiv(2 \pi)^{2} \delta_{\mathrm{D}}\left(\mathbf{l}_{1}+\mathbf{l}_{2}+\mathbf{l}_{3}+\mathbf{l}_{4}\right) T^{i}\left(\mathbf{l}_{1}, \mathbf{l}_{2}, \mathbf{l}_{3}, \mathbf{l}_{4}\right),
\end{aligned}
$$

where the angle brackets denote ensemble averages over possible realizations of the primordial CMB, large-scale structure between the observer and the surface of last-scattering, and instrumental noise. The subscript $c$ denotes the connected part of the four-point function and the superscript $i$ denotes the temperature map being considered $\left(\Theta^{\mathrm{t}}, \tilde{\Theta}\right.$, or $\left.\Theta^{\mathrm{n}}\right)$. The lensing-potential power spectrum can be defined analogously,

$$
\left\langle\phi(\mathbf{l}) \phi\left(\mathbf{l}^{\prime}\right)\right\rangle_{\mathrm{LSS}}=(2 \pi)^{2} \delta_{D}\left(\mathbf{l}+\mathbf{l}^{\prime}\right) C_{l}^{\phi \phi},
$$

where here the angle brackets denote an average over all realizations of the large-scale structure. We make the assumption that primordial fluctuations at the last-scattering surface are Gaussian. Gaussian statistics are fully described by a power spectrum; the Gaussian four-point correlator, $\left\langle\Theta\left(\mathbf{l}_{1}\right) \ldots \Theta\left(\mathbf{l}_{4}\right)\right\rangle_{c}$ is zero. The instrumental noise $\Theta^{\mathrm{n}}$ is also assumed to be Gaussian, as is the lensing potential $\phi$. This second assumption is justified because the dominant contributions to the lensing potential come from intermediate redshifts $1 \lesssim z \lesssim 3$ at which linear theory holds.

Using these definitions, we can calculate the anticipated power spectrum and trispectrum of the observed CMB map. Because the instrumental noise is uncorrelated with the signal, the power spectrum of the observed map is the sum of signal and noise power spectra,

$$
C_{l}^{\Theta \Theta \mathrm{t}}=\tilde{C}_{l}^{\Theta \Theta}+C_{l}^{\Theta \Theta \mathrm{n}} .
$$

The power spectrum of the noise component is given by:

$$
C_{l}^{\Theta \Theta \mathrm{n}}=f_{\mathrm{sky}} w^{-1} e^{l^{2} \sigma_{b}^{2}},
$$

where $f_{\text {sky }}$ is the fraction of the sky surveyed, $w^{-1}$ is the variance per unit area on the sky, and $\sigma_{b}=\theta / \sqrt{8 \ln 2}$ is the effective beamwidth of the instrument expressed in terms of its full-width half-maximum resolution $\theta$. A CMB experiment that spends a time $t_{\text {pix }}$ examining each of $N_{\text {pix }}$ pixels with detectors of sensitivity $s$ will have a variance per unit area $w^{-1}=4 \pi\left(s / T_{\mathrm{CMB}}\right)^{2} /\left(t_{\text {pix }} N_{\text {pix }}\right)$ [12]. The power spectrum of the lensed CMB can be determined by inserting Eq. (2) into Eq. (4) as discussed in [8],

$$
\tilde{C}_{l}^{\Theta \Theta}=\left[1-\int \frac{d^{2} \mathbf{l}_{1}}{(2 \pi)^{2}} C_{l_{1}}^{\phi \phi}\left(\mathbf{l}_{1} \cdot \mathbf{l}\right)^{2}\right] C_{l}^{\Theta \Theta}+\int \frac{d^{2} \mathbf{l}_{1}}{(2 \pi)^{2}} C_{\left|\mathbf{l}-\mathbf{l}_{1}\right|}^{\Theta \Theta} C_{l_{1}}^{\phi \phi}\left[\left(\mathbf{l}-\mathbf{l}_{1}\right) \cdot \mathbf{l}_{1}\right]^{2} .
$$

This result is given to linear order in the lensing-potential power spectrum $C_{l}^{\phi \phi}$. Lensing neither creates nor destroys power in the CMB, but merely shifts the scales on which it occurs as seen by the fact that

$$
\tilde{\sigma}^{2}=\int \frac{d^{2} \mathbf{l}}{(2 \pi)^{2}} \tilde{C}_{l}^{\Theta \Theta}=\int \frac{d^{2} \mathbf{l}}{(2 \pi)^{2}} C_{l}^{\Theta \Theta}=\sigma^{2} .
$$

The observed CMB trispectrum can be calculated in a similar manner; under our assumptions of Gaussian instrumental noise and no secondary anisotropies the trispectrum of the lensed component $\tilde{\Theta}$ is the sole contribution to the total observed trispectrum,

$$
\begin{aligned}
T^{\mathrm{t}}\left(\mathbf{l}_{1}, \mathbf{l}_{2}, \mathbf{l}_{3}, \mathbf{l}_{4}\right) & =\tilde{T}^{\Theta}\left(\mathbf{l}_{1}, \mathbf{l}_{2}, \mathbf{l}_{3}, \mathbf{l}_{4}\right) \\
& =-C_{l_{3}}^{\Theta \Theta} C_{l_{4}}^{\Theta \Theta}\left[C_{\left|\mathbf{l}_{1}+\mathbf{l}_{3}\right|}^{\phi \phi}\left[\left(\mathbf{l}_{1}+\mathbf{l}_{3}\right) \cdot \mathbf{l}_{3}\right]\left[\left(\mathbf{l}_{1}+\mathbf{l}_{3}\right) \cdot \mathbf{l}_{4}\right]+C_{\left|\mathbf{l}_{2}+\mathbf{l}_{3}\right|}^{\phi \phi}\left[\left(\mathbf{l}_{2}+\mathbf{l}_{3}\right) \cdot \mathbf{l}_{3}\right]\left[\left(\mathbf{l}_{2}+\mathbf{l}_{3}\right) \cdot \mathbf{l}_{4}\right]\right]+\text { Perm. . }
\end{aligned}
$$

The term shown above is manifestly symmetric under the interchange $\mathbf{l}_{1} \leftrightarrow \mathbf{l}_{2}$, while the "+ Perm." represents five additional terms identical in form but with the replacement of $\left(\mathbf{l}_{1}, \mathbf{l}_{2}\right)$ and $\left(\mathbf{l}_{3}, \mathbf{l}_{4}\right)$ with the other five combinations of pairs. The total trispectrum is symmetric under the interchange of any given pair as one would expect. Having established a formalism within which to analyze weak lensing, we now consider the problem of reconstructing the lensing potential from an observed CMB temperature map. 


\section{LENSING-POTENTIAL ESTIMATORS}

In this Section, we examine lensing reconstruction following the approach of Ref. [6], largely adopting their notation as well. The only important difference in notation is that we use $\tilde{\Theta}$ to denote the lensed temperature field and $\Theta$ for the unlensed field following Ref. [8] and most recent papers. Ref. [6] uses the opposite convention. For $\mathbf{l} \neq-\mathbf{l}^{\prime}$ and to linear order in $\phi$,

$$
\left\langle\Theta^{\mathrm{t}}(\mathbf{l}) \Theta^{\mathrm{t}}\left(\mathbf{l}^{\prime}\right)\right\rangle_{\mathrm{CMB}}=f_{\Theta \Theta}\left(\mathbf{l}, \mathbf{l}^{\prime}\right) \phi(\mathbf{L}),
$$

where

$$
f_{\Theta \Theta}\left(\mathbf{l}, \mathbf{l}^{\prime}\right) \equiv C_{l}^{\Theta \Theta}(\mathbf{L} \cdot \mathbf{l})+C_{l^{\prime}}^{\Theta \Theta}\left(\mathbf{L} \cdot \mathbf{l}^{\prime}\right),
$$

and $\mathbf{L}=\mathbf{l}+\mathbf{l}^{\prime}$. Note that \langle\rangle$_{\mathrm{CMB}}$ differs from the unmarked \langle\rangle that first appeared in Eq. (4) in that it denotes an ensemble average only over different Gaussian realizations of the primordial CMB and instrument noise; a fixed realization of the large-scale structure is assumed. For the purposes of estimating the large-scale structure actually realized in our observable universe, this is the appropriate average to take to ensure that our estimators are truly unbiased for a typical realization of the primordial CMB. When calculating the noise associated with lensing-potential estimators and again for power spectrum estimation in $\S \mathrm{IV}$, we will return to the full unmarked ensemble average. Eq. (11), an immediate consquence of Eq. (2), suggests that a temperature-squared map appropriately filtered in Fourier space can serve as an estimator for the deflection field $\mathbf{d}(\mathbf{L}) \equiv i \mathbf{L} \phi(\mathbf{L})$. Hu and Okamoto define five different estimators for the deflection field constructed from various combinations of the temperature and polarization; we discuss the temperature-squared estimator in this Section and relegate the analogous formulae for polarization estimators to the Appendix. The minimum-variance temperature-squared estimator derived in Ref. [6] is

$$
\mathbf{d}_{\Theta \Theta}(\mathbf{L}) \equiv \frac{i \mathbf{L} A_{\Theta \Theta}(L)}{L^{2}} \int \frac{d^{2} \mathbf{l}_{1}}{(2 \pi)^{2}} \Theta^{\mathrm{t}}\left(\mathbf{l}_{1}\right) \Theta^{\mathrm{t}}\left(\mathbf{l}_{2}\right) F_{\Theta \Theta}\left(\mathbf{l}_{1}, \mathbf{l}_{2}\right)
$$

where

$$
\begin{gathered}
F_{\Theta \Theta}\left(\mathbf{l}_{1}, \mathbf{l}_{2}\right) \equiv \frac{f_{\Theta \Theta}\left(\mathbf{l}_{1}, \mathbf{l}_{2}\right)}{2 C_{l_{1}}^{\Theta \Theta t} C_{l_{2}}^{\Theta \Theta t}} \\
A_{\Theta \Theta}(L) \equiv L^{2}\left[\int \frac{d^{2} \mathbf{l}_{1}}{(2 \pi)^{2}} f_{\Theta \Theta}\left(\mathbf{l}_{1}, \mathbf{l}_{2}\right) F_{\Theta \Theta}\left(\mathbf{l}_{1}, \mathbf{l}_{2}\right)\right]^{-1},
\end{gathered}
$$

and $\mathbf{l}_{2}=\mathbf{L}-\mathbf{l}_{1}$. Substitution of Eqs. (11), (12), (14), and (15) into Eq. (13) shows the desired result,

$$
\left\langle\mathbf{d}_{\Theta \Theta}(\mathbf{L})\right\rangle_{\mathrm{CMB}}=\mathbf{d}(\mathbf{L}),
$$

namely that $\mathbf{d}_{\Theta \Theta}(\mathbf{L})$ is indeed an unbiased estimator for the deflection field in Fourier space. We now proceed to calculate the variance of this estimator. At first, we assume a complete knowledge of all lensing modes not examined by this estimator. In that case, we find

$$
\begin{aligned}
& \left\langle\mathbf{d}_{\Theta \Theta}^{*}(\mathbf{L}) \cdot \mathbf{d}_{\Theta \Theta}\left(\mathbf{L}^{\prime}\right)\right\rangle_{\mathrm{CMB}}-\left\langle\mathbf{d}_{\Theta \Theta}^{*}(\mathbf{L})\right\rangle_{\mathrm{CMB}} \cdot\left\langle\mathbf{d}_{\Theta \Theta}\left(\mathbf{L}^{\prime}\right)\right\rangle_{\mathrm{CMB}}=\left(\mathbf{L} \cdot \mathbf{L}^{\prime}\right) \frac{A_{\Theta \Theta}(L)}{L^{2}} \frac{A_{\Theta \Theta}\left(L^{\prime}\right)}{L^{\prime 2}} \\
& \quad \times \int \frac{d^{2} \mathbf{l}_{1}}{(2 \pi)^{2}} \int \frac{d^{2} \mathbf{l}_{1}^{\prime}}{(2 \pi)^{2}}\left\langle\Theta^{\mathrm{t}}\left(-\mathbf{l}_{1}\right) \Theta^{\mathrm{t}}\left(-\mathbf{l}_{2}\right) \Theta^{\mathrm{t}}\left(\mathbf{l}_{1}{ }^{\prime}\right) \Theta^{\mathrm{t}}\left(\mathbf{l}_{2}{ }^{\prime}\right)\right\rangle_{\mathrm{CMB}} F_{\Theta \Theta}\left(\mathbf{l}_{1}, \mathbf{l}_{2}\right) F_{\Theta \Theta}\left(\mathbf{l}_{1}{ }^{\prime}, \mathbf{l}_{2}{ }^{\prime}\right)-\mathbf{d}^{*}(\mathbf{L}) \cdot \mathbf{d}\left(\mathbf{L}^{\prime}\right),
\end{aligned}
$$

where $\mathbf{l}_{2}{ }^{\prime}=\mathbf{L}^{\prime}-\mathbf{l}_{1}{ }^{\prime}$. Evaluating the four-point function in the integrand of Eq. (17) to second order in the lensing field, we obtain 

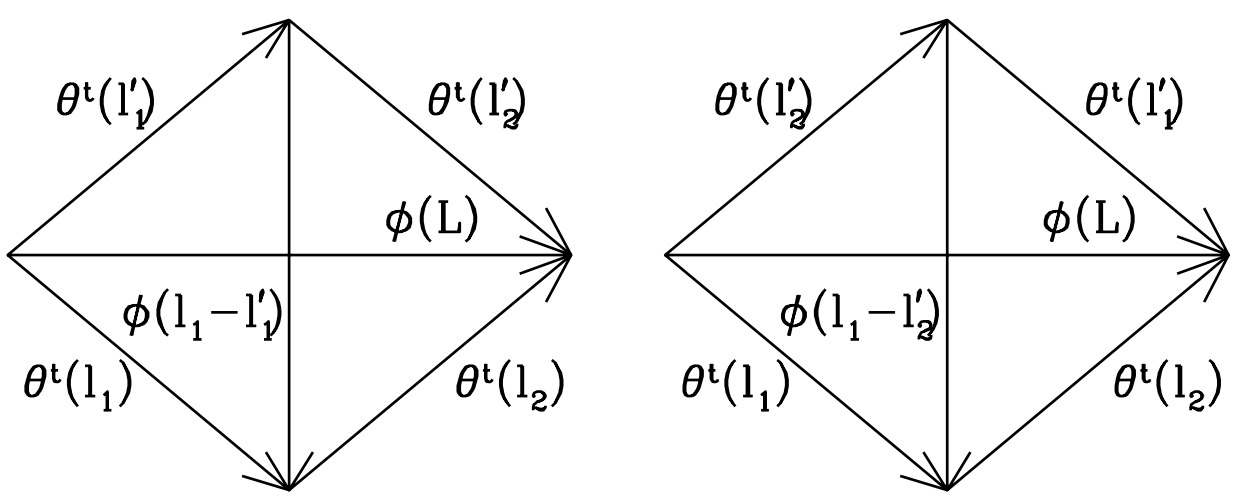

FIG. 1. The two quadrilaterals consistent with the constraint $\mathbf{L}-\mathbf{L}^{\prime}=\mathbf{l}_{1}+\mathbf{l}_{2}-\mathbf{l}_{1}{ }^{\prime}-\mathbf{l}_{2}{ }^{\prime}=0$ for the variance of the estimator $\mathbf{d}_{\Theta \Theta}(\mathbf{L})$. The lensing modes $\phi(\mathbf{L}), \phi\left(\mathbf{l}_{1}-\mathbf{l}_{1}{ }^{\prime}\right)$, and $\phi\left(\mathbf{l}_{1}-\mathbf{l}_{2}{ }^{\prime}\right)$, depicted as diagonals in the above quadrilaterals, induce non-Gaussian couplings between the modes of the observed temperature map represented as sides of these quadrilaterals. They lead to the three groups of linear terms appearing in Eq. (18).

$$
\begin{aligned}
& \left\langle\Theta^{\mathrm{t}}\left(-\mathbf{l}_{1}\right) \Theta^{\mathrm{t}}\left(-\mathbf{l}_{2}\right) \Theta^{\mathrm{t}}\left(\mathbf{l}_{1}{ }^{\prime}\right) \Theta^{\mathrm{t}}\left(\mathbf{l}_{2}{ }^{\prime}\right)\right\rangle_{\mathrm{CMB}}= \\
& \quad\left[\left(C_{l_{1}}^{\Theta \Theta}+C_{l_{1}}^{\Theta \Theta \mathrm{n}}\right)(2 \pi)^{2} \delta_{\mathrm{D}}(\mathbf{L})+\phi(-\mathbf{L}) f_{\Theta \Theta}\left(\mathbf{l}_{1}, \mathbf{l}_{2}\right)-\int \frac{d^{2} \mathbf{l}^{\prime}}{(2 \pi)^{2}} C_{l^{\prime}}^{\Theta \Theta} \phi\left(-\mathbf{l}_{1}-\mathbf{l}^{\prime}\right) \phi\left(-\mathbf{l}_{2}+\mathbf{l}^{\prime}\right)\left[\mathbf{l}^{\prime} \cdot\left(\mathbf{l}_{1}+\mathbf{l}^{\prime}\right)\right]\right. \\
& \left.\times\left[\mathbf{l}^{\prime} \cdot\left(\mathbf{l}_{2}-\mathbf{l}^{\prime}\right)\right]-\frac{1}{2} \int \frac{d^{2} \mathbf{l}^{\prime}}{(2 \pi)^{2}} \phi\left(\mathbf{l}^{\prime}\right) \phi\left(-\mathbf{L}-\mathbf{l}^{\prime}\right)\left\{C_{l_{1}}^{\Theta \Theta}\left(\mathbf{l}_{1} \cdot \mathbf{l}^{\prime}\right)\left[\mathbf{l}_{1} \cdot\left(\mathbf{L}+\mathbf{l}^{\prime}\right)\right]+C_{l_{2}}^{\Theta \Theta}\left(\mathbf{l}_{2} \cdot \mathbf{l}^{\prime}\right)\left[\mathbf{l}_{2} \cdot\left(\mathbf{L}+\mathbf{l}^{\prime}\right)\right]\right\}\right] \\
& \times\left[\left(C_{l_{1}^{\prime}}^{\Theta \Theta}+C_{l_{1}^{\prime}}^{\Theta \Theta \mathrm{n}}\right)(2 \pi)^{2} \delta_{\mathrm{D}}\left(\mathbf{L}^{\prime}\right)+\phi\left(\mathbf{L}^{\prime}\right) f_{\Theta \Theta}\left(\mathbf{l}_{1}{ }^{\prime}, \mathbf{l}_{2}{ }^{\prime}\right)-\int \frac{d^{2} \mathbf{l}^{\prime}}{(2 \pi)^{2}} C_{l^{\prime}}^{\Theta \Theta} \phi\left(\mathbf{l}_{1}{ }^{\prime}-\mathbf{l}^{\prime}\right) \phi\left(\mathbf{l}_{2}{ }^{\prime}+\mathbf{l}^{\prime}\right)\left[\mathbf{l}^{\prime} \cdot\left(\mathbf{l}_{1}{ }^{\prime}-\mathbf{l}^{\prime}\right)\right]\right. \\
& \left.\quad \times\left[\mathbf{l}^{\prime} \cdot\left(\mathbf{l}_{2}{ }^{\prime}+\mathbf{l}^{\prime}\right)\right]+\frac{1}{2} \int \frac{d^{2} \mathbf{l}^{\prime}}{(2 \pi)^{2}} \phi\left(\mathbf{l}^{\prime}\right) \phi\left(\mathbf{L}^{\prime}-\mathbf{l}^{\prime}\right)\left\{C_{l_{1}^{\prime}}^{\Theta \Theta}\left(\mathbf{l}_{1}{ }^{\prime} \cdot \mathbf{l}^{\prime}\right)\left[\mathbf{l}^{\prime}{ }^{\prime} \cdot\left(\mathbf{l}^{\prime}-\mathbf{L}^{\prime}\right)\right]+C_{l_{2}^{\prime}}^{\Theta \Theta}\left(\mathbf{l}_{2}{ }^{\prime} \cdot \mathbf{l}^{\prime}\right)\left[\mathbf{l}_{2}{ }^{\prime} \cdot\left(\mathbf{l}^{\prime}-\mathbf{L}^{\prime}\right)\right]\right\}\right]+ \text { Perm. }
\end{aligned}
$$

The terms given explicitly in Eq. (18) correspond to the correlations between $\Theta^{\mathrm{t}}\left(-\mathbf{l}_{1}\right)$ and $\Theta^{\mathrm{t}}\left(-\mathbf{l}_{2}\right)$ and those between $\Theta^{\mathrm{t}}\left(\mathbf{l}_{1}{ }^{\prime}\right)$ and $\Theta^{\mathrm{t}}\left(\mathbf{l}_{2}{ }^{\prime}\right)$. The "+ Perm." stands for two additional terms, identical in form, arising from the pairings $\left\langle\Theta^{\mathrm{t}}\left(-\mathbf{l}_{1}\right) \Theta^{\mathrm{t}}\left(\mathbf{l}_{1}{ }^{\prime}\right)\right\rangle_{\mathrm{CMB}}\left\langle\Theta^{\mathrm{t}}\left(-\mathbf{l}_{2}\right) \Theta^{\mathrm{t}}\left(\mathbf{l}_{2}{ }^{\prime}\right)\right\rangle_{\mathrm{CMB}}$ and $\left\langle\Theta^{\mathrm{t}}\left(-\mathbf{l}_{1}\right) \Theta^{\mathrm{t}}\left(\mathbf{l}_{2}{ }^{\prime}\right)\right\rangle_{\mathrm{CMB}}\left\langle\Theta^{\mathrm{t}}\left(-\mathbf{l}_{2}\right) \Theta^{\mathrm{t}}\left(\mathbf{l}_{1}{ }^{\prime}\right)\right\rangle_{\mathrm{CMB}}$. This expression indicates how uncertainty in the $\mathrm{CMB}$ at the last-scattering surface propagates into uncertainty in lensing reconstruction for a particular realization $\phi(\mathbf{L})$ of the large-scale structure. To linear order in Eq. (18), correlations between the modes $\Theta^{\mathrm{t}}\left(-\mathbf{l}_{1}\right), \Theta^{\mathrm{t}}\left(-\mathbf{l}_{2}\right), \Theta^{\mathrm{t}}\left(\mathbf{l}_{1}{ }^{\prime}\right)$, and $\Theta^{\mathrm{t}}\left(\mathbf{l}_{1}{ }^{\prime}\right)$ are induced by those lensing modes whose wavevectors are the sums of any pair of wavevectors of these four modes. These lensing modes are precisely those forming the diagonals of the quadrilaterals depicted in Fig. 1. In practice, we do not know the large-scale structure between us and the last-scattering surface, so we assume a variance given by Eq. (5) with a model-dependent power spectrum $C_{L}^{\phi \phi}$. We must average Eq. (17) over different realizations of the large-scale structure (denoted by \langle\rangle$_{\text {LSS }}$ ) to obtain the total expected variance of our estimator,

$$
\begin{aligned}
& \left\langle\left\langle\mathbf{d}_{\Theta \Theta}^{*}(\mathbf{L}) \cdot \mathbf{d}_{\Theta \Theta}\left(\mathbf{L}^{\prime}\right)\right\rangle_{\mathrm{CMB}}-\left\langle\mathbf{d}_{\Theta \Theta}^{*}(\mathbf{L})\right\rangle_{\mathrm{CMB}} \cdot\left\langle\mathbf{d}_{\Theta \Theta}\left(\mathbf{L}^{\prime}\right)\right\rangle_{\mathrm{CMB}}\right\rangle_{\mathrm{LSS}}=\left(\mathbf{L} \cdot \mathbf{L}^{\prime}\right) \frac{A_{\Theta \Theta}(L)}{L^{2}} \frac{A_{\Theta \Theta}\left(L^{\prime}\right)}{L^{\prime 2}} \\
& \quad \times \int \frac{d^{2} \mathbf{l}_{1}}{(2 \pi)^{2}} \int \frac{d^{2} \mathbf{l}_{1}^{\prime}}{(2 \pi)^{2}}\left\langle\Theta^{\mathrm{t}}\left(-\mathbf{l}_{1}\right) \Theta^{\mathrm{t}}\left(-\mathbf{l}_{2}\right) \Theta^{\mathrm{t}}\left(\mathbf{l}_{1}{ }^{\prime}\right) \Theta^{\mathrm{t}}\left(\mathbf{l}_{2}{ }^{\prime}\right)\right\rangle F_{\Theta \Theta}\left(\mathbf{l}_{1}, \mathbf{l}_{2}\right) F_{\Theta \Theta}\left(\mathbf{l}_{1}{ }^{\prime}, \mathbf{l}{ }^{\prime}\right)-(2 \pi)^{2} \delta_{\mathrm{D}}\left(\mathbf{L}-\mathbf{L}^{\prime}\right) C_{L}^{d d},
\end{aligned}
$$

where

$$
\left\langle\mathbf{d}^{*}(\mathbf{L}) \cdot \mathbf{d}\left(\mathbf{L}^{\prime}\right)\right\rangle=(2 \pi)^{2} \delta_{\mathrm{D}}\left(\mathbf{L}-\mathbf{L}^{\prime}\right) C_{L}^{d d}=(2 \pi)^{2} \delta_{\mathrm{D}}\left(\mathbf{L}-\mathbf{L}^{\prime}\right) L^{2} C_{L}^{\phi \phi} .
$$

The assumption that the lensing potential is Gaussian imposes the constraint $\mathbf{L}-\mathbf{L}^{\prime}=\mathbf{l}_{1}+\mathbf{l}_{2}-\mathbf{l}_{1}{ }^{\prime}-\mathbf{l}_{2}{ }^{\prime}=0$ which closes the quadrilaterals of Fig. 1. The average of the four-point correlation function in Eq. (19) can be calculated 
by further averaging Eq. (18) over the large-scale structure. Terms linear in the lensing field vanish when averaged over different realizations of the large-scale structure. Quadratic terms in the lensing field arise as products either of two linear terms or of a zeroth and second-order term. Averages over the product of two linear terms produce the connected part of the four-point correlation function, the trispectrum defined in Eq. (4). Averages over the product of a zeroth and second-order term have no connected portion, but instead furnish an implicit dependence on $C_{L}^{\phi \phi}$ in the total observed power spectrum of Eq. (6). The final result of averaging over the large-scale structure can be expressed in terms of the observed power spectrum and trispectrum,

$$
\begin{aligned}
\left\langle\Theta^{\mathrm{t}}\left(-\mathbf{l}_{1}\right) \Theta^{\mathrm{t}}\left(-\mathbf{l}_{2}\right) \Theta^{\mathrm{t}}\left(\mathbf{l}_{1}{ }^{\prime}\right) \Theta^{\mathrm{t}}\left(\mathbf{l}_{2}{ }^{\prime}\right)\right\rangle=(2 \pi)^{4}[ & C_{l_{1}}^{\Theta \Theta \mathrm{t}} C_{l_{1}^{\prime}}^{\Theta \Theta \mathrm{t}} \delta_{\mathrm{D}}(\mathbf{L}) \delta_{\mathrm{D}}\left(\mathbf{L}^{\prime}\right)+C_{l_{1}}^{\Theta \Theta \mathrm{t}} C_{l_{2}}^{\Theta \Theta \mathrm{t}}\left\{\delta_{\mathrm{D}}\left(\mathbf{l}_{1}{ }^{\prime}-\mathbf{l}_{1}\right) \delta_{\mathrm{D}}\left(\mathbf{l}_{2}{ }^{\prime}-\mathbf{l}_{2}\right)\right. \\
& \left.\left.+\delta_{\mathrm{D}}\left(\mathbf{l}_{2}{ }^{\prime}-\mathbf{l}_{1}\right) \delta_{\mathrm{D}}\left(\mathbf{l}_{1}{ }^{\prime}-\mathbf{l}_{2}\right)\right\}+(2 \pi)^{-2} T^{\mathrm{t}}\left(-\mathbf{l}_{1},-\mathbf{l}_{2}, \mathbf{l}_{1}{ }^{\prime}, \mathbf{l}_{2}{ }^{\prime}\right) \delta_{\mathrm{D}}\left(\mathbf{L}-\mathbf{L}^{\prime}\right)\right],
\end{aligned}
$$

where the trispectrum can written in terms of $f_{\Theta \Theta}\left(\mathbf{l}_{1}, \mathbf{l}_{2}\right)$ as

$$
T^{\mathrm{t}}\left(\mathbf{l}_{1}, \mathbf{l}_{2}, \mathbf{l}_{3}, \mathbf{l}_{4}\right)=C_{\left|\mathbf{l}_{1}+\mathbf{l}_{2}\right|}^{\phi \phi} f_{\Theta \Theta}\left(\mathbf{l}_{1}, \mathbf{l}_{2}\right) f_{\Theta \Theta}\left(\mathbf{l}_{3}, \mathbf{l}_{4}\right)+C_{\left|\mathbf{l}_{1}+\mathbf{l}_{3}\right|}^{\phi \phi} f_{\Theta \Theta}\left(\mathbf{l}_{1}, \mathbf{l}_{3}\right) f_{\Theta \Theta}\left(\mathbf{l}_{2}, \mathbf{l}_{4}\right)+C_{\left|\mathbf{l}_{1}+\mathbf{l}_{4}\right|}^{\phi \phi} f_{\Theta \Theta}\left(\mathbf{l}_{1}, \mathbf{l}_{4}\right) f_{\Theta \Theta}\left(\mathbf{l}_{2}, \mathbf{l}_{3}\right) .
$$

This form of the trispectrum is consistent with that of Eq. (10) given directly in terms of power spectra. Since $\mathbf{L} \neq 0$, $\delta_{\mathrm{D}}(\mathbf{L})=0$ and the first term of Eq. (21) vanishes. The remaining two terms containing pairs of delta functions, inserted into Eq. (19), yield the dominant contribution to the variance,

$$
\left\langle\left\langle\mathbf{d}_{\Theta \Theta}^{*}(\mathbf{L}) \cdot \mathbf{d}_{\Theta \Theta}\left(\mathbf{L}^{\prime}\right)\right\rangle_{\mathrm{CMB}}-\left\langle\mathbf{d}_{\Theta \Theta}^{*}(\mathbf{L})\right\rangle_{\mathrm{CMB}} \cdot\left\langle\mathbf{d}_{\Theta \Theta}\left(\mathbf{L}^{\prime}\right)\right\rangle_{\mathrm{CMB}}\right\rangle_{\mathrm{LSS}}=(2 \pi)^{2} \delta_{\mathrm{D}}\left(\mathbf{L}-\mathbf{L}^{\prime}\right)\left[N_{\Theta \Theta}^{(0)}, \Theta \Theta(L)+\ldots\right],
$$

where $N_{\Theta \Theta, \Theta \Theta}^{(0)}(L)=A_{\Theta \Theta}(L)$. Notice that $N_{\Theta \Theta, \Theta \Theta}^{(0)}(L)$ is zeroth order in the lensing potential $\phi$; it depends on the lensing potential power spectrum $C_{l}^{\phi \phi}$ only implicitly though the total observed power spectrum $C_{l}^{\Theta \Theta t}$. The ellipsis represents terms of higher order in $C_{l}^{\phi \phi}$ that we now proceed to calculate. These terms arise from the trispectrum term of Eq. (21) after $\left\langle\left\langle\mathbf{d}_{\Theta \Theta}^{*}(\mathbf{L})\right\rangle_{\mathrm{CMB}} \cdot\left\langle\mathbf{d}_{\Theta \Theta}\left(\mathbf{L}^{\prime}\right)\right\rangle_{\mathrm{CMB}}\right\rangle_{\mathrm{LSS}}=(2 \pi)^{2} \delta_{\mathrm{D}}\left(\mathbf{L}-\mathbf{L}^{\prime}\right) C_{L}^{d d}$ is removed. Substituting these results into Eq. (19), we find that to first order in $C_{l}^{\phi \phi}$,

$$
\left\langle\left\langle\mathbf{d}_{\Theta \Theta}^{*}(\mathbf{L}) \cdot \mathbf{d}_{\Theta \Theta}\left(\mathbf{L}^{\prime}\right)\right\rangle_{\mathrm{CMB}}-\left\langle\mathbf{d}_{\Theta \Theta}^{*}(\mathbf{L})\right\rangle_{\mathrm{CMB}} \cdot\left\langle\mathbf{d}_{\Theta \Theta}\left(\mathbf{L}^{\prime}\right)\right\rangle_{\mathrm{CMB}}\right\rangle_{\mathrm{LSS}}=(2 \pi)^{2} \delta_{\mathrm{D}}\left(\mathbf{L}-\mathbf{L}^{\prime}\right)\left[N_{\Theta \Theta, \Theta \Theta}^{(0)}(L)+N_{\Theta \Theta, \Theta \Theta}^{(1)}(L)\right],
$$

where $N_{\Theta \Theta, \Theta \Theta}^{(1)}(L)$ is given by,

$$
\begin{aligned}
& N_{\Theta \Theta, \Theta \Theta}^{(1)}(L)=\frac{A_{\Theta \Theta}^{2}(L)}{L^{2}} \int \frac{d^{2} \mathbf{l}_{1}}{(2 \pi)^{2}} \int \frac{d^{2} \mathbf{l}_{1}^{\prime}}{(2 \pi)^{2}} F_{\Theta \Theta}\left(\mathbf{l}_{1}, \mathbf{l}_{2}\right) F_{\Theta \Theta}\left(\mathbf{l}_{1}{ }^{\prime}, \mathbf{l}_{2}{ }^{\prime}\right) \\
& \quad \times\left\{C_{\left|\mathbf{l}_{1}-\mathbf{l}_{1}{ }^{\prime}\right|}^{\phi \phi} f_{\Theta \Theta}\left(-\mathbf{l}_{1}, \mathbf{l}_{1}{ }^{\prime}\right) f_{\Theta \Theta}\left(-\mathbf{l}_{2}, \mathbf{l}_{2}{ }^{\prime}\right)+C_{\left|\mathbf{l}_{1}-\mathbf{l}_{2}{ }^{\prime}\right|}^{\phi \phi} f_{\Theta \Theta}\left(-\mathbf{l}_{1}, \mathbf{l}_{2}{ }^{\prime}\right) f_{\Theta \Theta}\left(-\mathbf{l}_{2}, \mathbf{l}_{1}{ }^{\prime}\right)\right\} .
\end{aligned}
$$

The first-order contribution to the noise $N_{\Theta \Theta, \Theta \Theta}^{(1)}(L)$ involves integrals over the lensing-potential power spectrum, and thus probes lensing modes with wave vectors different from that of the estimator $\mathbf{d}_{\Theta \Theta}(\mathbf{L})$. It can be interpreted physically as interference from these other modes in the determination of the mode $\mathbf{d}(\mathbf{L})$ being estimated. The filter $F_{\Theta \Theta}\left(\mathbf{l}_{1}, \mathbf{l}_{2}\right)$ was chosen to optimize the signal-to-noise ratio in the absence of the first-order contribution $N_{\Theta \Theta, \Theta \Theta}^{(1)}(L)$; it is no longer an optimal filter once this additional noise is taken into account. As long as $N_{\Theta \Theta, \Theta \Theta}^{(1)}(L) \ll N_{\Theta \Theta}^{(0), \Theta \Theta}(L)$, the noise reduction that can be attained by re-optimizing our filter will not be significant. Formulas analagous to those presented here relevant to the construction of estimators using polarization data are given in the Appendix.

The significance of $N_{\Theta \Theta, \Theta \Theta}^{(1)}(L)$ for two different experiments is shown in Fig. 2 using the currently favored $\Lambda$ CDM cosmological model with baryon density $\Omega_{b}=0.05$, matter density $\Omega_{m}=0.35$, cosmological constant density $\Omega_{\Lambda}=$ 0.65 , Hubble parameter $h=0.65$, and power-spectrum amplitude $\sigma_{8}=0.9$. The Planck experiment is equivalent to a one-year, full-sky survey with temperature and polarization sensitivities of 12.42 and $26.02 \mu \mathrm{K} \sqrt{\sec }$ respectively and resolution $\theta=7.0$ arcminutes as described in $\S$ II. The reference experiment has the same resolution but superior sensitivities of 0.46 and $0.65 \mu \mathrm{K} \sqrt{\mathrm{sec}}$ for temperature and polarization. These estimates of experimental parameters are identical to those given for the Planck and reference experiments of Ref. [6]. The $\Theta E$ and $E E$ estimators have noise power spectra intermediate to those of the $\Theta \Theta$ and $E B$ estimators, while the $\Theta B$ estimator has substantially higher 


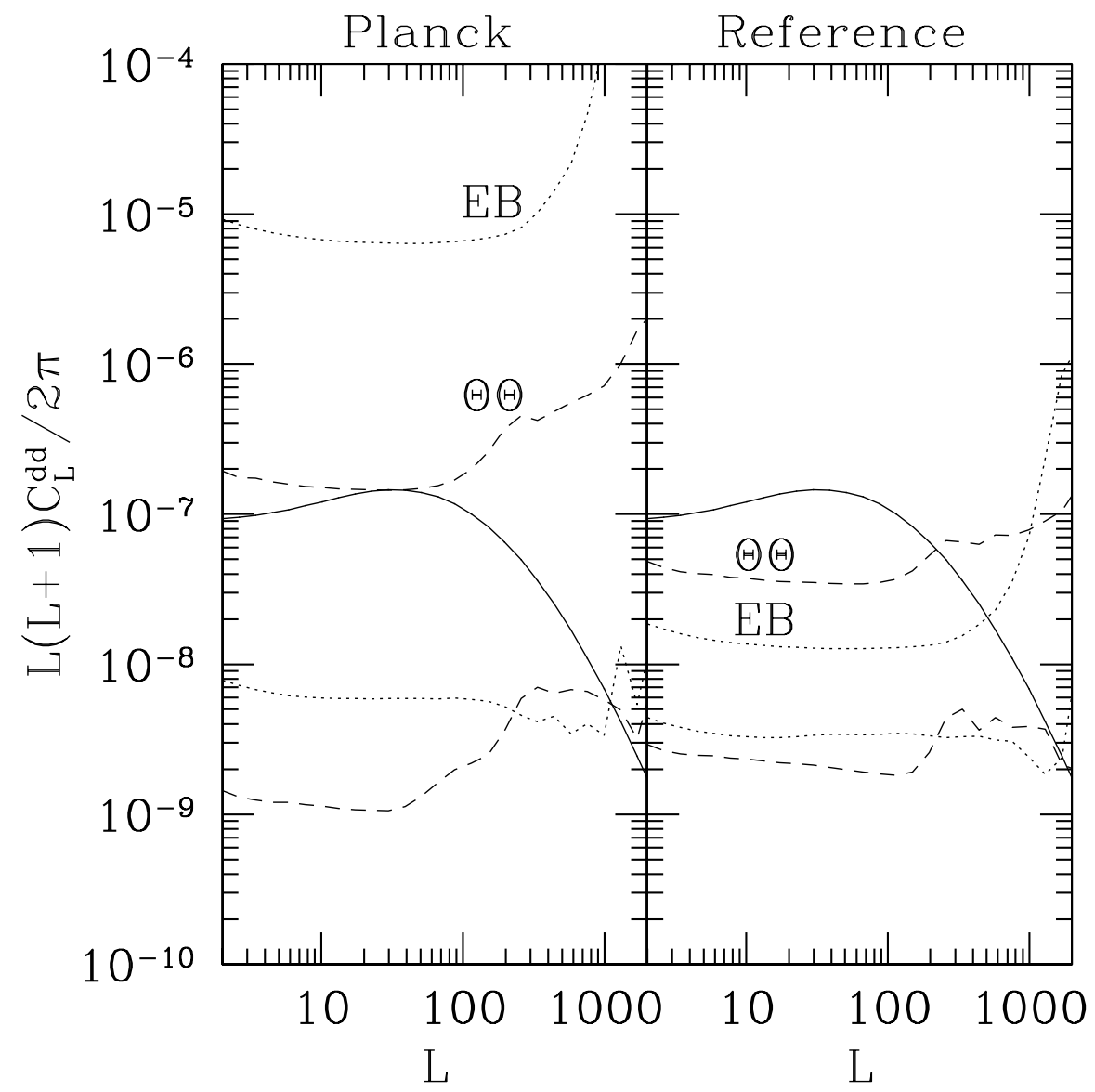

FIG. 2. Variances with which individual modes $\mathbf{d}(\mathbf{L})$ of the deflection field can be reconstructed by the Planck and reference experiments described in the text. The solid curves are the power spectra $C_{L}^{d d}$ anticipated for our $\Lambda$ CDM cosmological model. The upper and lower dashed curves are the zeroth and first-order noise power spectra $N_{\Theta \Theta, \Theta \Theta}^{(0)}(L)$ and $N_{\Theta \Theta, \Theta \Theta}^{(1)}(L)$ respectively for the temperature-based estimator $\mathbf{d}_{\Theta \Theta}(\mathbf{L})$, while the dotted curves are the corresponding noise variances for $\mathbf{d}_{E B}(\mathbf{L})$. A mode $\mathbf{d}(\mathbf{L})$ cannot be reconstructed with signal-to-noise greater than unity when $C_{L}^{d d} \leq N_{\Theta \Theta, \Theta \Theta}^{(0)}(L)+N_{\Theta \Theta, \Theta \Theta}^{(1)}(L)$.

noise because the primordial CMB lacks true B-modes in the absence of inflationary gravitational waves. We see that for Planck, with its comparatively inferior polarization sensitivity, the $\Theta \Theta$ estimator will be best although it will be unable to detect individual Fourier modes of the deflection field at the $1 \sigma$ level. The reference experiment, and further experiments with similar sensitivity and even higher resolution, should be able to push $1 \sigma$ detection of individual $\mathbf{d}(\mathbf{L})$ modes to $L \simeq 1000$ by primarily relying on the $E B$ estimator. In these cases the secondary noise $N_{E B, E B}^{(1)}(L)$ is only smaller than the dominant noise $N_{E B, E B}^{(0)}(L)$ by a factor of a few, whereas for higher sensitivity (noisier) experiments like Planck it is smaller by at least an order-of-magnitude. This illustrates an interesting point, apparent from Fig. 2, that the zeroth-order noise $N_{\Theta \Theta, \Theta \Theta}^{(0)}(L)$ declines dramatically with decreasing sensitivity until it becomes dominated by cosmic variance while the $N_{\Theta \Theta, \Theta \Theta}^{(1)}(L)$ is largely unaffected by instrument sensitivity. The reasons for this trend are that instrument noise appears in $N_{\Theta \Theta, \Theta \Theta}^{(0)}(L)=A_{\Theta \Theta}(L)$ through its contribution to the denominator of $F_{\Theta \Theta}\left(\mathbf{l}_{1}, \mathbf{l}_{2}\right)$ as shown by Eqs. (14) and (15). Decreasing instrument noise raises the value of $F_{\Theta \Theta}\left(\mathbf{l}_{1}, \mathbf{l}_{2}\right)$ thereby lowering $N_{\Theta \Theta, \Theta \Theta}^{(0)}(L)$. By contrast instrument noise is reflected in $N_{\Theta \Theta, \Theta \Theta}^{(1)}(L)$ through its effects on both $A_{\Theta \Theta}(L)$ and $F_{\Theta \Theta}\left(\mathbf{l}_{1}, \mathbf{l}_{2}\right)$ as shown in Eq. (25). Smaller instrument noise raises $F_{\Theta \Theta}\left(\mathbf{l}_{1}, \mathbf{l}_{2}\right)$ as before, driving $N_{\Theta \Theta, \Theta \Theta}^{(1)}(L)$ up in this case, but this is compensated by a decrease in $A_{\Theta \Theta}(L)$ which appears as a prefactor outside the integrals. These two effects largely cancel each other out, rendering $N_{\Theta \Theta, \Theta \Theta}^{(1)}(L)$ remarkably insensitive to instrument noise. 


\section{POWER SPECTRUM ESTIMATION}

Although complete reconstruction of the deflection field $\mathbf{d}(\mathbf{L})$ can be an enormously powerful tool, such as for B-mode subtraction [4], for some purposes estimates of the lensing-potential power spectrum $C_{L}^{\phi \phi}$ are sufficient. This power spectrum is a model-dependent prediction of theories of large-scale structure formation, and therefore estimates of the power spectrum from real data could be used to test these theories as well as the consistency of other determinations of cosmological parameters. Furthermore, since estimates of all the modes $\mathbf{d}(\mathbf{L})$ with $|\mathbf{L}|=L$ can be

combined to estimate $C_{L}^{\phi \phi}, 1 \sigma$ detection of the power spectrum can be pushed to much higher $L$ than can that of individual modes. The deflection-field estimator $\mathbf{d}_{\Theta \Theta}(\mathbf{L})$ derived in the preceding Section can be used to construct an estimator for $C_{L}^{\phi \phi}$. Our first guess for an appropriate lensing-potential power spectrum estimator is

$$
D_{L} \equiv \frac{(2 \pi)^{2}}{A L^{2}} \frac{1}{2 \pi L \Delta L} \int_{a_{L}} \frac{d^{2} \mathbf{l}}{(2 \pi)^{2}} \mathbf{d}_{\Theta \Theta}(\mathbf{l}) \cdot \mathbf{d}_{\Theta \Theta}(-\mathbf{l})
$$

where $A$ is the area of the sky surveyed and $a_{L}$ is an annulus of radius $L$ and width $\Delta L$. We ensemble average our estimator over different realizations of the CMB and large-scale structure using Eq. (24) by bringing $\left\langle\left\langle\mathbf{d}_{\Theta \Theta}^{*}(\mathbf{L})\right\rangle_{\mathrm{CMB}}\right.$. $\left.\left\langle\mathbf{d}_{\Theta \Theta}\left(\mathbf{L}^{\prime}\right)\right\rangle_{\mathrm{CMB}}\right\rangle_{\mathrm{LSS}}$ from the left to the right-hand side. This yields

$$
\left\langle D_{L}\right\rangle=\frac{(2 \pi)^{2}}{A L^{2}} \frac{1}{2 \pi L \Delta L} \delta_{\mathrm{D}}(0) \int_{a_{L}} d^{2} \mathbf{l}\left[l^{2} C_{l}^{\phi \phi}+N_{\Theta \Theta, \Theta \Theta}^{(0)}(l)+N_{\Theta \Theta, \Theta \Theta}^{(1)}(l)\right] .
$$

The definition of the Dirac delta function,

$$
(2 \pi)^{2} \delta_{\mathrm{D}}(\mathbf{l}) \equiv \int_{A} d \hat{\mathbf{n}} e^{i \mathbf{l} \cdot \hat{\mathbf{n}}}
$$

implies that $\delta_{\mathrm{D}}(0)=A /(2 \pi)^{2}$. Furthermore, in the limit that $\Delta L$ is small compared to the scales on which $l^{2} C_{l}^{\phi \phi}+$ $N_{\Theta \Theta, \Theta \Theta}^{(0)}(l)+N_{\Theta \Theta, \Theta \Theta}^{(1)}(l)$ is varying, we can evaluate the integrand of Eq. (27) at its central value $l=L$ and extract it from the integral. The integral over the annulus $a_{L}$ cancels the factor $2 \pi L \Delta L$ in the denominator, reducing Eq. (27) to

$$
\left\langle D_{L}\right\rangle=C_{L}^{\phi \phi}+L^{-2}\left[N_{\Theta \Theta, \Theta \Theta}^{(0)}(L)+N_{\Theta \Theta, \Theta \Theta}^{(1)}(L)\right] .
$$

$D_{L}$ is indeed an estimator for the lensing-potential power spectrum $C_{L}^{\phi \phi}$, albeit a biaed one. Note that the bias in the power-spectrum estimator $D_{L}$ is precisely the same as the variance shown in Eq. (24) with which we were able to determine each individual lensing mode. This is no coincidence; it reflects the fact that there are no grounds $a$ priori on which to differentiate the variance with which we can reconstruct individual modes $\mathbf{d}(\mathbf{L})$ from the intrinsic variance $C_{L}^{d d}$ of the underlying distribution from which they are drawn. To obtain an unbiased estimator to compare with theoretical predictions, we subtract off this unwanted reconstruction variance,

$$
\widehat{C}_{L}^{\phi \phi} \equiv D_{L}-L^{-2}\left[N_{\Theta \Theta, \Theta \Theta}^{(0)}(L)+N_{\Theta \Theta, \Theta \Theta}^{(1)}(L)\right] .
$$

Since $N_{\Theta \Theta, \Theta \Theta}^{(1)}(L)$ as defined in Eq. (25) itself depends on $C_{L}^{\phi \phi}$, this subtraction and evaluation must be performed iteratively until a self-consistent solution is obtained. The variance of our estimator $\widehat{C}_{L}^{\phi \phi}$ can be calculated in the usual manner,

$$
\sigma_{\widehat{C}_{L}^{\phi \phi}}^{2} \equiv\left\langle\left(\widehat{C}_{L}^{\phi \phi}\right)^{2}\right\rangle-\left\langle\widehat{C}_{L}^{\phi \phi}\right\rangle^{2}=\left\langle\left(D_{L}\right)^{2}\right\rangle-\left\langle D_{L}\right\rangle^{2}
$$

Evaluating this expression requires us to calculate

$$
\left\langle\left(D_{L}\right)^{2}\right\rangle=\frac{(2 \pi)^{4}}{A^{2} L^{4}} \frac{1}{(2 \pi L \Delta L)^{2}} \int_{a_{L}} \frac{d^{2} \mathbf{l}_{1}{ }^{\prime}}{(2 \pi)^{2}} \int_{a_{L}} \frac{d^{2} \mathbf{l}_{2}{ }^{\prime}}{(2 \pi)^{2}}\left\langle\left[\mathbf{d}_{\Theta \Theta}\left(\mathbf{l}_{1}{ }^{\prime}\right) \cdot \mathbf{d}_{\Theta \Theta}\left(-\mathbf{l}_{1}{ }^{\prime}\right)\right]\left[\mathbf{d}_{\Theta \Theta}\left(\mathbf{l}_{2}{ }^{\prime}\right) \cdot \mathbf{d}_{\Theta \Theta}\left(-\mathbf{l}_{2}{ }^{\prime}\right)\right]\right\rangle .
$$

Since $\mathbf{d}_{\Theta \Theta}(\mathbf{L})$ is a quadratic estimator in the temperature map, Eq. (32) includes the following integral over the eight-point correlation function in Fourier space, 


$$
\begin{aligned}
& \left\langle\left[\mathbf{d}_{\Theta \Theta}\left(\mathbf{l}_{1}{ }^{\prime}\right) \cdot \mathbf{d}_{\Theta \Theta}\left(-\mathbf{l}_{1}{ }^{\prime}\right)\right]\left[\mathbf{d}_{\Theta \Theta}\left(\mathbf{l}_{2}{ }^{\prime}\right) \cdot \mathbf{d}_{\Theta \Theta}\left(-\mathbf{l}_{2}{ }^{\prime}\right)\right]\right\rangle=\frac{A_{\Theta \Theta}^{2}\left(l_{1}^{\prime}\right) A_{\Theta \Theta}^{2}\left(l_{2}^{\prime}\right)}{\left(l_{1}^{\prime}\right)^{2}\left(l_{2}^{\prime}\right)^{2}} \int \frac{d^{2} \mathbf{k}_{1}}{(2 \pi)^{2}} \int \frac{d^{2} \mathbf{k}_{3}}{(2 \pi)^{2}} \int \frac{d^{2} \mathbf{k}_{5}}{(2 \pi)^{2}} \int \frac{d^{2} \mathbf{k}_{7}}{(2 \pi)^{2}} \\
& \quad \times\left\{F_{\Theta \Theta}\left(\mathbf{k}_{1}, \mathbf{k}_{2}\right) F_{\Theta \Theta}\left(\mathbf{k}_{3}, \mathbf{k}_{4}\right) F_{\Theta \Theta}\left(\mathbf{k}_{5}, \mathbf{k}_{6}\right) F_{\Theta \Theta}\left(\mathbf{k}_{7}, \mathbf{k}_{8}\right)\left\langle\Theta^{\mathrm{t}}\left(\mathbf{k}_{1}\right) \Theta^{\mathrm{t}}\left(\mathbf{k}_{2}\right) \Theta^{\mathrm{t}}\left(\mathbf{k}_{3}\right) \Theta^{\mathrm{t}}\left(\mathbf{k}_{4}\right) \Theta^{\mathrm{t}}\left(\mathbf{k}_{5}\right) \Theta^{\mathrm{t}}\left(\mathbf{k}_{6}\right) \Theta^{\mathrm{t}}\left(\mathbf{k}_{7}\right) \Theta^{\mathrm{t}}\left(\mathbf{k}_{8}\right)\right\rangle\right\},
\end{aligned}
$$

where $\mathbf{k}_{2}=\mathbf{l}_{1}{ }^{\prime}-\mathbf{k}_{1}, \mathbf{k}_{4}=-\mathbf{l}_{1}{ }^{\prime}-\mathbf{k}_{3}, \mathbf{k}_{6}=\mathbf{l}_{2}{ }^{\prime}-\mathbf{k}_{5}$, and $\mathbf{k}_{8}=-\mathbf{l}_{2}{ }^{\prime}-\mathbf{k}_{7}$. A fully general eight-point correlation function consists of a connected part, as well as terms proportional to the product of lower-order correlation functions. Under the assumption that both the primordial CMB and the lensing potential are governed by Gaussian statistics, all correlation functions higher than the four-point have vanishing connected parts [13]. The temperature eight-point correlation function will therefore be composed of three groups of terms; membership in a group being determined by whether the term contains zero, one, or two factors of the trispectrum. Since the trispectrum given in Eq. (10) is first order in the lensing-potential power spectrum $C_{L}^{\phi \phi}$, terms of these three groups are zeroth, first, and second order respectively in $C_{L}^{\phi \phi}$. Combinatorics determines the number of terms in each group. There are: $\frac{1}{4 !}\left(\begin{array}{l}8 \\ 2\end{array}\right)\left(\begin{array}{l}6 \\ 2\end{array}\right)\left(\begin{array}{l}4 \\ 2\end{array}\right)\left(\begin{array}{l}2 \\ 2\end{array}\right)=$ 105 different ways of dividing $\left(\mathbf{k}_{1}, \ldots, \mathbf{k}_{8}\right)$ into four pairs, and hence there will be 105 terms in the group containing no trispectra. Similar calculations reveal that there are $\frac{1}{2 !}\left(\begin{array}{l}8 \\ 4\end{array}\right)\left(\begin{array}{l}4 \\ 2\end{array}\right)\left(\begin{array}{l}2 \\ 2\end{array}\right)=210$ terms in the second group and $\frac{1}{2 !}\left(\begin{array}{l}8 \\ 4\end{array}\right)\left(\begin{array}{l}4 \\ 4\end{array}\right)=35$ terms in the third group. Many terms in all three groups will vanish for the same reason that the first term vanished in the four-point correlation function of Eq. (21); these terms are proportional to a Dirac delta function evaluated at nonzero argument. Consider now the first group of terms, those that are zeroth order in $C_{L}^{\phi \phi}$. The 60 nonvanishing terms in this group each contain four Dirac delta functions; they can be further segregated into the 12 terms that allow two of the integrals over $\mathbf{k}_{i}$ appearing in Eq. (33) to be immediately evaluated via Dirac delta functions, and the 48 terms that allow evaluation of three $\mathbf{k}_{i}$ integrals. The first 12 terms, inserted into Eq. (33) and appropriately evaluated using the normalization of Eq. (15), yield

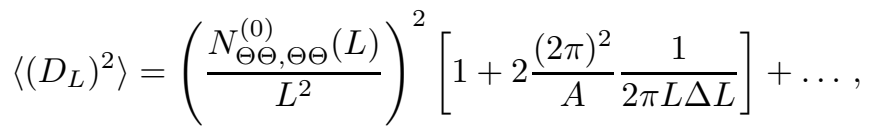

while the remaining 48 terms give the final result to zeroth order in $C_{L}^{\phi \phi}$,

$$
\begin{aligned}
\left\langle\left(D_{L}\right)^{2}\right\rangle= & \left(\frac{N_{\Theta \Theta, \Theta \Theta}^{(0)}(L)}{L^{2}}\right)^{2}\left[1+2 \frac{(2 \pi)^{2}}{A} \frac{1}{2 \pi L \Delta L}\right] \\
& +\frac{(2 \pi)^{2}}{A L^{4}} \frac{2}{(2 \pi L \Delta L)^{2}} \int_{a_{L}} \frac{d^{2} \mathbf{l}_{1}{ }^{\prime}}{(2 \pi)^{2}} \int_{a_{L}} \frac{d^{2} \mathbf{l}_{2}{ }^{\prime}}{(2 \pi)^{2}} \frac{A_{\Theta \Theta}^{2}\left(l_{1}^{\prime}\right) A_{\Theta \Theta}^{2}\left(l_{2}^{\prime}\right)}{\left(l_{1}^{\prime}\right)^{2}\left(l_{2}^{\prime}\right)^{2}} \int \frac{d^{2} \mathbf{k}_{1}}{(2 \pi)^{2}} f_{\Theta \Theta}\left(\mathbf{k}_{1}, \mathbf{k}_{2}\right) P\left(\mathbf{k}_{1}, \mathbf{k}_{2}, \mathbf{l}_{1}{ }^{\prime}, \mathbf{l}_{2}{ }^{\prime}\right)
\end{aligned}
$$

where

$$
\begin{aligned}
P\left(\mathbf{k}_{1}, \mathbf{k}_{2}, \mathbf{l}_{1}{ }^{\prime}, \mathbf{l}_{2}{ }^{\prime}\right)= & f_{\Theta \Theta}\left(-\mathbf{l}_{2}{ }^{\prime}-\mathbf{k}_{1}, \mathbf{l}_{2}{ }^{\prime}-\mathbf{k}_{2}\right) F_{\Theta \Theta}\left(-\mathbf{k}_{1}, \mathbf{l}_{2}{ }^{\prime}+\mathbf{k}_{1}\right) F_{\Theta \Theta}\left(-\mathbf{k}_{2},-\mathbf{l}_{2}{ }^{\prime}+\mathbf{k}_{2}\right) \\
& +f_{\Theta \Theta}\left(\mathbf{l}_{2}{ }^{\prime}-\mathbf{k}_{1},-\mathbf{l}_{2}{ }^{\prime}-\mathbf{k}_{2}\right) F_{\Theta \Theta}\left(-\mathbf{k}_{1},-\mathbf{l}_{2}{ }^{\prime}+\mathbf{k}_{1}\right) F_{\Theta \Theta}\left(-\mathbf{k}_{2}, \mathbf{l}_{2}{ }^{\prime}+\mathbf{k}_{2}\right) \\
& +f_{\Theta \Theta}\left(-\mathbf{l}_{2}{ }^{\prime}-\mathbf{k}_{1}, \mathbf{l}_{1}{ }^{\prime}-\mathbf{k}_{2}\right) F_{\Theta \Theta}\left(-\mathbf{k}_{1}, \mathbf{l}_{2}{ }^{\prime}+\mathbf{k}_{1}\right) F_{\Theta \Theta}\left(-\mathbf{k}_{2},-\mathbf{l}_{1}{ }^{\prime}+\mathbf{k}_{2}\right) \\
& +f_{\Theta \Theta}\left(\mathbf{l}_{1}{ }^{\prime}-\mathbf{k}_{1},-\mathbf{l}_{2}{ }^{\prime}-\mathbf{k}_{2}\right) F_{\Theta \Theta}\left(-\mathbf{k}_{1},-\mathbf{l}_{1}{ }^{\prime}+\mathbf{k}_{1}\right) F_{\Theta \Theta}\left(-\mathbf{k}_{2}, \mathbf{l}_{2}{ }^{\prime}+\mathbf{k}_{2}\right) \\
& +f_{\Theta \Theta}\left(\mathbf{l}_{1}{ }^{\prime}-\mathbf{k}_{1}, \mathbf{l}_{2}{ }^{\prime}-\mathbf{k}_{2}\right) F_{\Theta \Theta}\left(-\mathbf{k}_{1},-\mathbf{l}_{1}{ }^{\prime}+\mathbf{k}_{1}\right) F_{\Theta \Theta}\left(-\mathbf{k}_{2},-\mathbf{l}_{2}{ }^{\prime}+\mathbf{k}_{2}\right) \\
& +f_{\Theta \Theta}\left(\mathbf{l}_{2}{ }^{\prime}-\mathbf{k}_{1}, \mathbf{l}_{1}{ }^{\prime}-\mathbf{k}_{2}\right) F_{\Theta \Theta}\left(-\mathbf{k}_{1},-\mathbf{l}_{2}{ }^{\prime}+\mathbf{k}_{1}\right) F_{\Theta \Theta}\left(-\mathbf{k}_{2},-\mathbf{l}_{1}{ }^{\prime}+\mathbf{k}_{2}\right) .
\end{aligned}
$$

When $\left\langle D_{L}\right\rangle^{2}$ is subtracted from $\left\langle\left(D_{L}\right)^{2}\right\rangle$ in Eq. (31), the first term in the square brackets of Eq. (35) will be eliminated. Minimizing the variance associated with this estimator then consists of making an optimal choice of $\Delta L(L)$. The first noise term is proportional to $\frac{(2 \pi)^{2} / A}{2 \pi L \Delta L}$. For a survey of area $A,(2 \pi)^{2} / A$ is the specific area of an individual mode in $\mathbf{L}$ space and $2 \pi L \Delta L$ is the area in $\mathbf{L}$ space over which the power-spectrum estimator takes an average. This ratio is therefore the inverse of the number of individual $\mathbf{d}_{\Theta \Theta}(\mathbf{L})$ modes whose inverse variances are added to determine 
the inverse variance of $\widehat{C}_{L}^{\phi \phi}$. It is obviously minimized by choosing $(2 \pi)^{2} / A \ll 2 \pi L \Delta L(L)$. The second term, that involving $P\left(\mathbf{k}_{1}, \mathbf{k}_{2}, \mathbf{l}_{1}{ }^{\prime}, \mathbf{l}_{2}{ }^{\prime}\right)$, differs from the first noise term in that a Dirac delta function has been used to evaluate an additional $\mathbf{k}_{i}$ integral rather than an annulus integral. Since the integrands are of the same order, we expect the second noise term to be suppressed relative to the first by a factor $2 \pi L \Delta L(L) / \pi l_{\max }^{2}$ where $l_{\max } \simeq \pi / \theta$ is set by the resolution $\theta$ of the survey. Under the conservative assumption $L \Delta L(L) \ll 1 / \theta^{2}$, namely that we are probing scales well above our resolution, this term is assured to be small. We neglect such terms for the remainder of this paper. If we insert the portions of the eight-point correlation function that are first and second order in $C_{L}^{\phi \phi}$ into Eq. (33) and evaluate using Eq. (25), we find

$$
\left\langle\left(D_{L}\right)^{2}\right\rangle=L^{-4}\left(C_{L}^{d d}+N_{\Theta \Theta, \Theta \Theta}^{(0)}(L)+N_{\Theta \Theta, \Theta \Theta}^{(1)}(L)\right)^{2}\left[1+2 \frac{(2 \pi)^{2}}{A} \frac{1}{2 \pi L \Delta L}\right]
$$

and

$$
\sigma_{\widehat{C}_{L}^{\phi \phi}}^{2}=2 \frac{(2 \pi)^{2}}{A} \frac{1}{2 \pi L \Delta L} L^{-4}\left(C_{L}^{d d}+N_{\Theta \Theta, \Theta \Theta}^{(0)}(L)+N_{\Theta \Theta, \Theta \Theta}^{(1)}(L)\right)^{2} .
$$

This result agrees with that given in Ref. [6] after subtracting our newly derived term $N_{\Theta \Theta, \Theta \Theta}^{(1)}(L)$.

The term $N_{\Theta \Theta, \Theta \Theta}^{(1)}(L)$ and corresponding terms for polarization-based estimators have two principal effects on power spectrum estimation. As shown in Eq. (38), they provide a fractional contribution to the variance of roughly $2 N_{\Theta \Theta, \Theta \Theta}^{(1)}(L) / C_{L}^{d d}$ when $C_{L}^{d d}$ dominates the variance as in the reference experiment in the right-hand panel of Fig. 2. For the $\Lambda \mathrm{CDM}$ cosmological model considered here this represents an increase of $5-15 \%$ in the variance of the $E B$ estimator for $L \lesssim 1000$. More importantly, $N_{\Theta \Theta \Theta \Theta \Theta}^{(1)}(L)$ acts as a bias for the naive estimator $D_{L}$ as shown by Eq. (29). If this bias is not calculated and subtracted iteratively to form the unbiased estimator $\widehat{C}_{L}^{\phi \phi}$ as in Eq. (30), $C_{L}^{d d}$ will be systematically overestimated by $5-10 \%$ at low $L$ and by increasingly larger amounts at $L \gtrsim 100$ as the signal $L(L+1) C_{L}^{d d} / 2 \pi$ begins to plummet while $N_{\Theta \Theta, \Theta \Theta}^{(1)}(L)$ remains comparatively flat.

Having evaluated the variance of our estimator $\widehat{C}_{L}^{\phi \phi}$, we consider whether this estimator has a substantial covariance

$$
\sigma_{\widehat{C}_{L}^{\phi \phi} \widehat{C}_{L^{\prime}}^{\phi \phi}} \equiv\left\langle\left(\widehat{C}_{L}^{\phi \phi}-C_{L}^{\phi \phi}\right)\left(\widehat{C}_{L^{\prime}}^{\phi \phi}-C_{L^{\prime}}^{\phi \phi}\right)\right\rangle=\left\langle D_{L} D_{L^{\prime}}\right\rangle-\left\langle D_{L}\right\rangle\left\langle D_{L^{\prime}}\right\rangle .
$$

The estimator $D_{L}$ as defined in Eq. (26) implies that:

$$
\left\langle D_{L} D_{L^{\prime}}\right\rangle=\left(\frac{2 \pi}{A L L^{\prime}}\right)^{2} \frac{1}{L \Delta L L^{\prime} \Delta L^{\prime}} \int_{a_{L}} \frac{d^{2} \mathbf{l}_{1}{ }^{\prime}}{(2 \pi)^{2}} \int_{a_{L^{\prime}}} \frac{d^{2} \mathbf{l}_{2}{ }^{\prime}}{(2 \pi)^{2}}\left\langle\left[\mathbf{d}_{\Theta \Theta}\left(\mathbf{l}_{1}{ }^{\prime}\right) \cdot \mathbf{d}_{\Theta \Theta}\left(-\mathbf{l}_{1}{ }^{\prime}\right)\right]\left[\mathbf{d}_{\Theta \Theta}\left(\mathbf{l}_{2}{ }^{\prime}\right) \cdot \mathbf{d}_{\Theta \Theta}\left(-\mathbf{l}_{2}{ }^{\prime}\right)\right]\right\rangle
$$

which can be evaluated using the same integral over the eight-point correlation function described in Eq. (33). Whereas 60 of the 105 zeroth-order terms in $C_{L}^{\phi \phi}$ coming from this equation were nonvanishing for the variance, for the covariance only 52 terms are nonzero provided that the widths $\Delta L$ and $\Delta L^{\prime}$ are chosen so that the annuli $a_{L}$ and $a_{L^{\prime}}$ do not overlap. This leads to a result analogous to Eq. (35),

$$
\begin{aligned}
\left\langle D_{L} D_{L^{\prime}}\right\rangle=\frac{N_{\Theta \Theta, \Theta \Theta}^{(0)}(L)}{L^{2}} \frac{N_{\Theta \Theta, \Theta \Theta}^{(0)}\left(L^{\prime}\right)}{L^{\prime 2}}+ \\
\frac{2}{A L^{3} \Delta L L^{\prime 3} \Delta L^{\prime}} \int_{a_{L}} \frac{d^{2} \mathbf{l}_{1}{ }^{\prime}}{(2 \pi)^{2}} \int_{a_{L^{\prime}}} \frac{d^{2} \mathbf{l}_{2}{ }^{\prime}}{(2 \pi)^{2}} \frac{A_{\Theta \Theta}^{2}\left(l_{1}^{\prime}\right) A_{\Theta \Theta}^{2}\left(l_{2}^{\prime}\right)}{\left(l_{1}^{\prime}\right)^{2}\left(l_{2}^{\prime}\right)^{2}} \int \frac{d^{2} \mathbf{k}_{1}}{(2 \pi)^{2}} f_{\Theta \Theta}\left(\mathbf{k}_{1}, \mathbf{k}_{2}\right) P\left(\mathbf{k}_{1}, \mathbf{k}_{2}, \mathbf{l}_{1}{ }^{\prime}, \mathbf{l}_{2}{ }^{\prime}\right) .
\end{aligned}
$$

Note that the eight terms missing from the covariance when compared to the variance have altered the first term of Eq. (41), and that it was precisely these terms that provided the dominant contribution to the variance of Eq. (38) when $\Delta L(L)$ was chosen appropriately. We therefore find that to zeroth order in $C_{L}^{\phi \phi}$, the covariance is given by

$$
\sigma_{\widehat{C}_{L}^{\phi \phi} \widehat{C}_{L^{\prime}}^{\phi \phi}}=\frac{2}{A L \Delta L L^{\prime} \Delta L^{\prime}}\left(\frac{N_{\Theta \Theta}^{(0)}, \Theta \Theta}{L^{2}}(L) \frac{N_{\Theta \Theta, \Theta \Theta}^{(0)}\left(L^{\prime}\right)}{L^{\prime 2}}\right)^{2} \int_{a_{L}} \frac{d^{2} \mathbf{l}_{1}{ }^{\prime}}{(2 \pi)^{2}} \int_{a_{L^{\prime}}} \frac{d^{2} \mathbf{l}_{2}{ }^{\prime}}{(2 \pi)^{2}} \int \frac{d^{2} \mathbf{k}_{1}}{(2 \pi)^{2}} f_{\Theta \Theta}\left(\mathbf{k}_{1}, \mathbf{k}_{2}\right) P\left(\mathbf{k}_{1}, \mathbf{k}_{2}, \mathbf{l}_{1}{ }^{\prime}, \mathbf{l}_{2}{ }^{\prime}\right),
$$




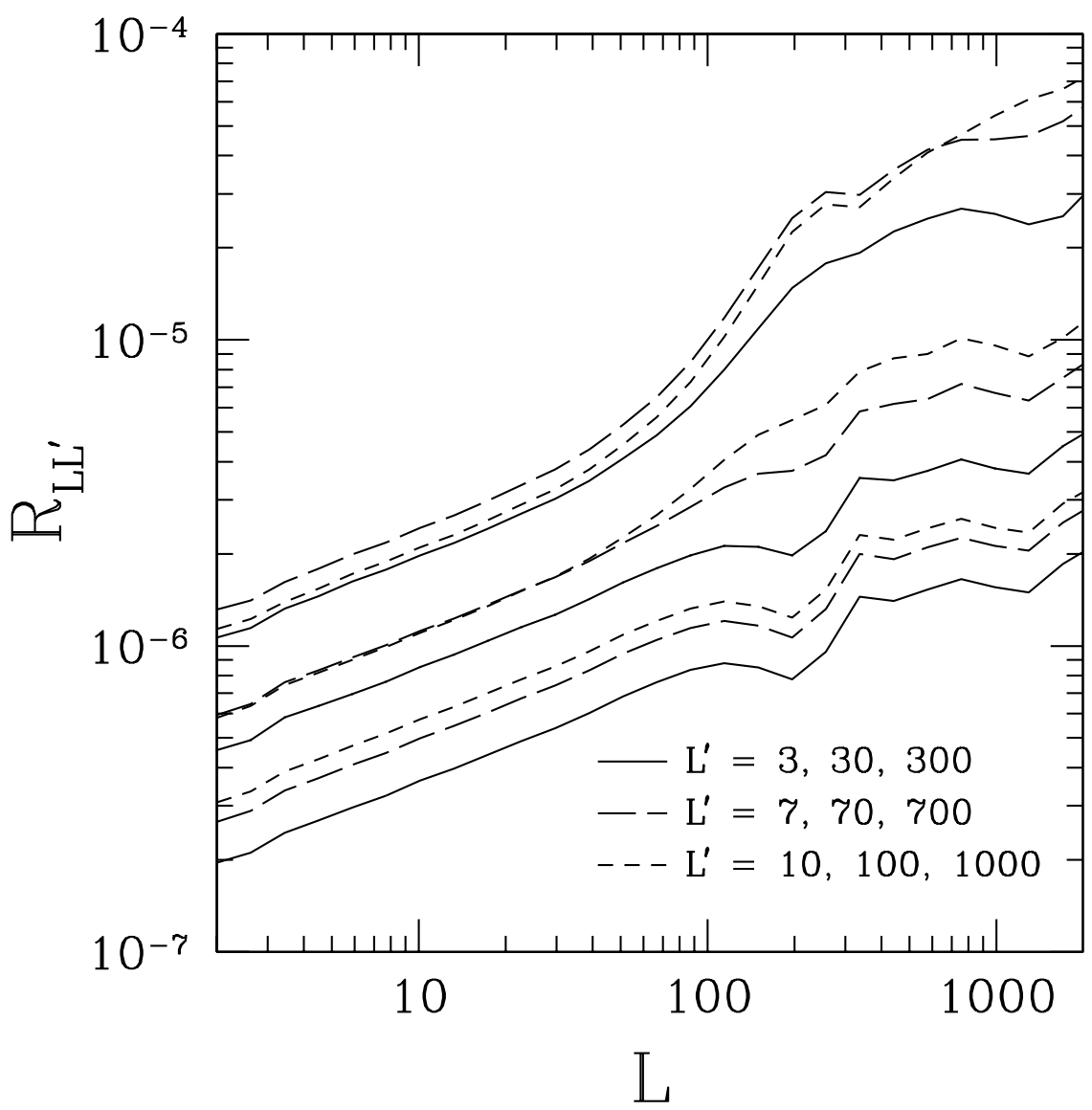

FIG. 3. The ratio $R_{L L^{\prime}}$ for Planck as a function of $L$ for fixed values of $L^{\prime}$. The solid curves correspond to $L^{\prime}=3,30,300$ ascending from bottom to top, while the long-dashed and short-dashed curves correspond to $L^{\prime}=7,70,700$ and $L^{\prime}=10,100,1000$ respectively, again with curves in each sequence appearing from bottom to top in the figure.

where we have extracted the $A_{\Theta \Theta}\left(l_{i}^{\prime}\right)$ from the annular integrals since they are slowly varying over the widths $\Delta L$ and $\Delta L^{\prime}$. For the same reasons that terms of this form were a subdominant contribution to the variance as discussed previously, we expect the covariance to be suppressed as well. If we define the ratio

$$
R_{L L^{\prime}} \equiv \frac{\sigma_{\widehat{C}_{L}^{\phi \phi} \widehat{C}_{L^{\prime}}^{\phi \phi}}}{\sqrt{\sigma_{\widehat{C}_{L}^{\phi \phi}}^{2} \sigma_{\widehat{C}_{L^{\prime}}^{\phi \phi}}^{2}}},
$$

we can quantify this suppression. The triple integral of Eq. (42), appearing in the numerator of $R_{L L^{\prime}}$, involves integration over annuli with radii $L$ and $L^{\prime}$ and one integration over all Fourier space. The triple integrals in the variances $\sigma_{\widehat{C}_{L}^{\phi \phi}}^{2}$ and $\sigma_{\widehat{C}_{L^{\prime}}^{\phi \phi}}^{2}$ appearing in the denominator of $R_{L L^{\prime}}$ each consist of a single integration over an annulus of radius $L$ and $L^{\prime}$ respectively and two integrations over all Fourier space. If we make the crude assumption that the integrand is constant, the ratio $R_{L L^{\prime}}$ will simply be the ratio of these areas,

$$
R_{L L^{\prime}} \simeq \sqrt{(2 \pi L \Delta L)\left(2 \pi L^{\prime} \Delta L^{\prime}\right)} / \pi l_{\max }^{2}
$$

The ratio $R_{L L^{\prime}}$ is evaluated numerically for Planck in Fig. 3 as a function of $L$ for various fixed values of $L^{\prime}$. The estimators $\widehat{C}_{L}^{\phi \phi}$ and $\widehat{C}_{L^{\prime}}^{\phi \phi}$ were chosen such that $\Delta L=\Delta L^{\prime}=1$, while integrals over Fourier space were cut off at $l_{\max }=5000$. Substituting these values into Eq. (44), we expect $R_{L L^{\prime}} \simeq 8.0 \times 10^{-8} \sqrt{L L^{\prime}}$. This crude estimate is surprisingly close to the numerically obtained results of Fig. 3; in particular the slope of the curves is approximately $1 / 2$ on this log-log plot. Even at $L \simeq 1000, R_{L L^{\prime}} \lesssim 4.0 \times 10^{-4}$ suggesting that covariance in power-spectrum estimation can safely be neglected for Planck. The estimate of Eq. (44) implicitly depends on the experimental resolution $\theta$ 
because the integrand appearing in expressions for the variance and covariance decreases rapidly for $L \gtrsim l_{\max } \simeq \pi / \theta$. For future experiments with better resolution than Planck, $l_{\max }$ will be higher implying by Eq. (44) that covariance will be even more negligible.

\section{DISCUSSION}

Weak gravitational lensing induces non-Gaussian correlations between modes of the observed CMB temperature map as shown in Eq. (11). These correlations, and assumptions about the Gaussian nature of the primordial CMB, can be used to construct several temperature and polarization-based estimators of the Fourier modes $\mathbf{d}(\mathbf{L})$ of the deflection field. This procedure was outlined in Ref. [6], however in calculating the noise associated with this reconstruction, an assumption was made that the observed temperature map was Gaussian. In the presence of lensing this assumption is invalid; when calculating the variance of quadratic estimators all permutations of the observed trispectrum must be taken into account. One such permutation reflects the desired correlation making our estimator sensitive to $\mathbf{d}(\mathbf{L})$, but the remaining two permutations induce additional variance proportional to the lensing-potential power spectrum $C_{L}^{\phi \phi}$. While subdominant, this variance will become increasing significant for future experiments as shown in the right-hand panel of Fig. 2. Since the power spectrum $C_{L}^{\phi \phi}$ is itself a measure of uncertainty in the deflection field, this additional variance in lensing reconstruction acts as a bias during power-spectrum estimation because there is no a priori way to distinguish it from the intrinsic variance of the underlying distribution. Our calculation of the dependence of this variance on $C_{L}^{\phi \phi}$ allows it in principle to be subtracted iteratively, which will prevent a systematic $5-10 \%$ overestimate of $C_{L}^{\phi \phi}$ at low $L$.

We close by considering several possible observational obstacles to the scheme for lensing reconstruction and powerspectrum estimation presented above. One hindrance is secondary contributions to the CMB such as the SZ and ISW effects. These effects increase the total temperature power spectrum appearing in the denominator of the optimum filter $F_{\Theta \Theta}\left(\mathbf{l}_{1}, \mathbf{l}_{2}\right)$ of Eq. (14) as would additional instrumental noise. They also correlate with the large-scale structure at low redshifts inducing further non-Gaussian couplings and additional variance to lensing reconstruction. Fortunately for our purposes the frequency dependence of the thermal SZ effect differs from that of a blackbody. It can therefore be separated in principle from the lensed primordial CMB by an experiment with several frequency channels [11]. The ISW effect cannot be removed in this manner, but is too small to significantly inhibit lensing reconstruction. Polarization-dependent secondary effects are expected to appear at higher orders in the density contrast [14], and we therefore anticipate that they will not make a contribution at the levels considered here. A potentially more serious problem is that of galactic foregrounds, which though uncorrelated with the lensing signal may be substantial at certain frequencies. Significant polarization has also been observed in some of these sources [15]. We hope to understand and minimize the effects of galactic foregrounds in future work, and to pursue further refinements of lensing reconstruction.

\section{ACKNOWLEDGMENTS}

We thank Wayne Hu for useful discussions. This work was supported in part by NASA NAG5-11985 and DoE DEFG03-92-ER40701. Kesden acknowledges the support of an NSF Graduate Fellowship and AC acknowledges support from the Sherman Fairchild Foundation.

\section{APPENDIX A: POLARIZATION-BASED ESTIMATORS}

Here we provide the appropriate formulas for deriving the variance associated with polarization-based estimators of the deflection field $\mathbf{d}(\mathbf{L})$. The CMB polarization can be decomposed into E and B-modes [16]. These modes are mixed by weak lensing such that to linear order in $\phi(\mathbf{L})$,

$$
\begin{aligned}
& \tilde{E}(\mathbf{l})=E(\mathbf{l})-\int \frac{d^{2} \mathbf{l}_{1}}{(2 \pi)^{2}}\left[E\left(\mathbf{l}_{1}\right) \cos 2\left(\varphi_{\mathbf{l}_{1}}-\varphi_{\mathbf{l}}\right)-B\left(\mathbf{l}_{1}\right) \sin 2\left(\varphi_{\mathbf{l}_{1}}-\varphi_{\mathbf{l}}\right)\right] \phi\left(\mathbf{l}-\mathbf{l}_{1}\right)\left[\left(\mathbf{l}-\mathbf{l}_{1}\right) \cdot \mathbf{l}_{1}\right], \\
& \tilde{B}(\mathbf{l})=B(\mathbf{l})-\int \frac{d^{2} \mathbf{l}_{1}}{(2 \pi)^{2}}\left[E\left(\mathbf{l}_{1}\right) \sin 2\left(\varphi_{\mathbf{l}_{1}}-\varphi_{\mathbf{l}}\right)+B\left(\mathbf{l}_{1}\right) \cos 2\left(\varphi_{\mathbf{l}_{1}}-\varphi_{\mathbf{l}}\right)\right] \phi\left(\mathbf{l}-\mathbf{l}_{1}\right)\left[\left(\mathbf{l}-\mathbf{l}_{1}\right) \cdot \mathbf{l}_{1}\right] .
\end{aligned}
$$

We can exploit the sensitivity of the polarization modes to the lensing potential to construct lensing estimators from quadratic combinations of polarization modes. We generalize Eq. (11) to arbitrary combinations $\left\{X, X^{\prime}\right\}$ of $\Theta, \mathrm{E}$, 
and B-modes as first derived in Ref. [6],

$$
\left\langle X^{\mathrm{t}}(\mathbf{l}) X^{\prime \mathrm{t}}\left(\mathbf{l}^{\prime}\right)\right\rangle_{\mathrm{CMB}}=f_{X X^{\prime}}\left(\mathbf{l}, \mathbf{l}^{\prime}\right) \phi(\mathbf{L}),
$$

where

$$
\begin{aligned}
& f_{\Theta E}\left(\mathbf{l}, \mathbf{l}^{\prime}\right)=C_{l}^{\Theta E} \cos 2\left(\varphi_{\mathbf{l}}-\varphi_{\mathbf{l}^{\prime}}\right)(\mathbf{L} \cdot \mathbf{l})+C_{l^{\prime}}^{\Theta E}\left(\mathbf{L} \cdot \mathbf{l}^{\prime}\right)=f_{E \Theta}\left(\mathbf{l}^{\prime}, \mathbf{l}\right), \\
& f_{\Theta B}\left(\mathbf{l}, \mathbf{l}^{\prime}\right)=C_{l}^{\Theta E} \sin 2\left(\varphi_{\mathbf{l}}-\varphi_{\mathbf{l}^{\prime}}\right)(\mathbf{L} \cdot \mathbf{l})=f_{B \Theta}\left(\mathbf{l}^{\prime}, \mathbf{l}\right), \\
& f_{E E}\left(\mathbf{l}, \mathbf{l}^{\prime}\right)=\left[C_{l}^{E E}(\mathbf{L} \cdot \mathbf{l})+C_{l^{\prime}}^{E E}\left(\mathbf{L} \cdot \mathbf{l}^{\prime}\right)\right] \cos 2\left(\varphi_{\mathbf{l}}-\varphi_{\mathbf{l}^{\prime}}\right), \\
& f_{E B}\left(\mathbf{l}, \mathbf{l}^{\prime}\right)=\left[C_{l}^{E E}(\mathbf{L} \cdot \mathbf{l})+C_{l^{\prime}}^{B B}\left(\mathbf{L} \cdot \mathbf{l}^{\prime}\right)\right] \sin 2\left(\varphi_{\mathbf{l}}-\varphi_{\mathbf{l}^{\prime}}\right)=f_{B E}\left(\mathbf{l}^{\prime}, \mathbf{l}\right), \\
& f_{B B}\left(\mathbf{l}, \mathbf{l}^{\prime}\right)=\left[C_{l}^{B B}(\mathbf{L} \cdot \mathbf{l})+C_{l^{\prime}}^{B B}\left(\mathbf{L} \cdot \mathbf{l}^{\prime}\right)\right] \cos 2\left(\varphi_{\mathbf{l}}-\varphi_{\mathbf{l}^{\prime}}\right) .
\end{aligned}
$$

In deriving these results we used parity considerations to demand $C_{l}^{\Theta B}=C_{l}^{E B}=0$. Using these relations, we follow the approach of Ref. [6] to derive symmetric lensing estimators

$$
\mathbf{d}_{X X}(\mathbf{L}) \equiv \frac{i \mathbf{L} A_{X X}(L)}{L^{2}} \int \frac{d^{2} \mathbf{l}_{1}}{(2 \pi)^{2}} X^{\mathrm{t}}\left(\mathbf{l}_{1}\right) X^{\mathrm{t}}\left(\mathbf{l}_{2}\right) F_{X X}\left(\mathbf{l}_{1}, \mathbf{l}_{2}\right),
$$

and

$$
\mathbf{d}_{X X^{\prime}}(\mathbf{L}) \equiv \frac{i \mathbf{L} A_{X X^{\prime}}(L)}{L^{2}} \int \frac{d^{2} \mathbf{l}_{1}}{(2 \pi)^{2}} \frac{1}{2}\left[X^{\mathrm{t}}\left(\mathbf{l}_{1}\right) X^{\prime \mathrm{t}}\left(\mathbf{l}_{2}\right)+X^{\prime \mathrm{t}}\left(\mathbf{l}_{1}\right) X^{\mathrm{t}}\left(\mathbf{l}_{2}\right)\right] F_{X X^{\prime}}\left(\mathbf{l}_{1}, \mathbf{l}_{2}\right) .
$$

We have explicitly symmetrized our estimators for $X \neq X^{\prime}$ to simplify the form of the optimal filter. The normalization bias of the estimators is removed by choosing

$$
A_{X X}(L) \equiv L^{2}\left[\int \frac{d^{2} \mathbf{l}_{1}}{(2 \pi)^{2}} f_{X X}\left(\mathbf{l}_{1}, \mathbf{l}_{2}\right) F_{X X}\left(\mathbf{l}_{1}, \mathbf{l}_{2}\right)\right]^{-1},
$$

and

$$
A_{X X^{\prime}}(L) \equiv L^{2}\left[\int \frac{d^{2} \mathbf{l}_{1}}{(2 \pi)^{2}} \frac{1}{2}\left[f_{X X^{\prime}}\left(\mathbf{l}_{1}, \mathbf{l}_{2}\right)+f_{X X^{\prime}}\left(\mathbf{l}_{2}, \mathbf{l}_{1}\right)\right] F_{X X^{\prime}}\left(\mathbf{l}_{1}, \mathbf{l}_{2}\right)\right]^{-1} .
$$

The minimum-variance filters $F_{X X^{\prime}}\left(\mathbf{l}_{1}, \mathbf{l}_{2}\right)$ for the various cases $\left\{X, X^{\prime}\right\}$ are given by

$$
\begin{aligned}
F_{\Theta E}\left(\mathbf{l}_{1}, \mathbf{l}_{2}\right) & =\frac{f_{\Theta E}\left(\mathbf{l}_{1}, \mathbf{l}_{2}\right)+f_{\Theta E}\left(\mathbf{l}_{2}, \mathbf{l}_{1}\right)}{C_{l_{1}}^{\Theta \Theta \mathrm{t}} C_{l_{2}}^{E E \mathrm{t}}+2 C_{l_{1}}^{\Theta E \mathrm{t}} C_{l_{2}}^{\Theta E \mathrm{t}}+C_{l_{1}}^{E E \mathrm{t}} C_{l_{2}}^{\Theta \Theta \mathrm{t}}}, \\
F_{\Theta B}\left(\mathbf{l}_{1}, \mathbf{l}_{2}\right) & =\frac{f_{\Theta B}\left(\mathbf{l}_{1}, \mathbf{l}_{2}\right)+f_{\Theta B}\left(\mathbf{l}_{2}, \mathbf{l}_{1}\right)}{C_{l_{1}}^{\Theta \Theta \mathrm{t}} C_{l_{2}}^{E E \mathrm{t}}+C_{l_{1}}^{E E \mathrm{t}} C_{l_{2}}^{\Theta \Theta \mathrm{t}}}, \\
F_{E E}\left(\mathbf{l}_{1}, \mathbf{l}_{2}\right) & =\frac{f_{E E}\left(\mathbf{l}_{1}, \mathbf{l}_{2}\right)}{2 C_{l_{1}}^{E E \mathrm{t}} C_{l_{2}}^{E E \mathrm{t}}}, \\
F_{E B}\left(\mathbf{l}_{1}, \mathbf{l}_{2}\right) & =\frac{f_{E B}\left(\mathbf{l}_{1}, \mathbf{l}_{2}\right)+f_{E B}\left(\mathbf{l}_{2}, \mathbf{l}_{1}\right)}{C_{l_{1}}^{E E \mathrm{t}} C_{l_{2}}^{B B \mathrm{t}}+C_{l_{1}}^{B B \mathrm{t}} C_{l_{2}}^{E E \mathrm{t}}}, \\
F_{B B}\left(\mathbf{l}_{1}, \mathbf{l}_{2}\right) & =\frac{f_{B B}\left(\mathbf{l}_{1}, \mathbf{l}_{2}\right)}{2 C_{l_{1}}^{B B \mathrm{t}} C_{l_{2}}^{B B \mathrm{t}}} .
\end{aligned}
$$

Using these optimal filters for the estimators defined in Eqs. (A4) and (A5), we can calculate the variances for these estimators in a fashion entirely analogoug to Eq. (19),

$$
\begin{aligned}
& \left\langle\left\langle\mathbf{d}_{X X}^{*}(\mathbf{L}) \cdot \mathbf{d}_{X X}\left(\mathbf{L}^{\prime}\right)\right\rangle_{\mathrm{CMB}}-\left\langle\mathbf{d}_{X X}^{*}(\mathbf{L})\right\rangle_{\mathrm{CMB}} \cdot\left\langle\mathbf{d}_{X X}\left(\mathbf{L}^{\prime}\right)\right\rangle_{\mathrm{CMB}}\right\rangle_{\mathrm{LSS}}=\frac{A_{X X}(L)}{L} \frac{A_{X X}\left(L^{\prime}\right)}{L^{\prime}} \\
& \quad \times \int \frac{d^{2} \mathbf{l}_{1}}{(2 \pi)^{2}} \int \frac{d^{2} \mathbf{l}_{1}^{\prime}}{(2 \pi)^{2}}\left\langle X^{\mathrm{t}}\left(-\mathbf{l}_{1}\right) X^{\mathrm{t}}\left(-\mathbf{l}_{2}\right) X^{\mathrm{t}}\left(\mathbf{l}_{1}{ }^{\prime}\right) X^{\mathrm{t}}\left(\mathbf{l}_{2}{ }^{\prime}\right)\right\rangle F_{X X}\left(\mathbf{l}_{1}, \mathbf{l}_{2}\right) F_{X X}\left(\mathbf{l}_{1}{ }^{\prime}, \mathbf{l}_{2}{ }^{\prime}\right)-(2 \pi)^{2} \delta_{\mathrm{D}}\left(\mathbf{L}-\mathbf{L}^{\prime}\right) C_{L}^{d d},
\end{aligned}
$$




$$
\begin{aligned}
& \left\langle\left\langle\mathbf{d}_{X X^{\prime}}^{*}(\mathbf{L}) \cdot \mathbf{d}_{X X^{\prime}}\left(\mathbf{L}^{\prime}\right)\right\rangle_{\mathrm{CMB}}-\left\langle\mathbf{d}_{X X^{\prime}}^{*}(\mathbf{L})\right\rangle_{\mathrm{CMB}} \cdot\left\langle\mathbf{d}_{X X^{\prime}}\left(\mathbf{L}^{\prime}\right)\right\rangle_{\mathrm{CMB}}\right\rangle_{\mathrm{LSS}}=\frac{A_{X X^{\prime}}(L)}{L} \frac{A_{X X^{\prime}}\left(L^{\prime}\right)}{L^{\prime}} \int \frac{d^{2} \mathbf{l}_{1}}{(2 \pi)^{2}} \int \frac{d^{2} \mathbf{l}_{1}^{\prime}}{(2 \pi)^{2}} \\
& \quad \times \frac{1}{4}\left\langle\left[X^{\mathrm{t}}\left(-\mathbf{l}_{1}\right) X^{\prime \mathrm{t}}\left(-\mathbf{l}_{2}\right)+X^{\prime \mathrm{t}}\left(-\mathbf{l}_{1}\right) X^{\mathrm{t}}\left(-\mathbf{l}_{2}\right)\right]\left[X^{\mathrm{t}}\left(\mathbf{l}_{1}{ }^{\prime}\right) X^{\prime \mathrm{t}}\left(\mathbf{l}_{2}{ }^{\prime}\right)+X^{\prime \mathrm{t}}\left(\mathbf{l}_{1}{ }^{\prime}\right) X^{\mathrm{t}}\left(\mathbf{l}_{2}{ }^{\prime}\right)\right]\right\rangle F_{X X^{\prime}}\left(\mathbf{l}_{1}, \mathbf{l}_{2}\right) F_{X X^{\prime}}\left(\mathbf{l}_{1}{ }^{\prime}, \mathbf{l}_{2}{ }^{\prime}\right) \\
& \quad-(2 \pi)^{2} \delta_{\mathrm{D}}\left(\mathbf{L}-\mathbf{L}^{\prime}\right) C_{L}^{d d} .
\end{aligned}
$$

As for that of the temperature estimator, these variance will consist of zeroth-order terms in $C_{L}^{\phi \phi}, N_{X X, X X}^{(0)}(L)=$ $A_{X X}(L)$ and $N_{X X^{\prime}, X X^{\prime}}^{(0)}(L)=A_{X X^{\prime}}(L)$, and first-order terms,

$$
\begin{aligned}
& N_{X X, X X}^{(1)}(L)=\frac{A_{X X}^{2}(L)}{L^{2}} \int \frac{d^{2} \mathbf{l}_{1}}{(2 \pi)^{2}} \int \frac{d^{2} \mathbf{l}_{1}^{\prime}}{(2 \pi)^{2}} F_{X X}\left(\mathbf{l}_{1}, \mathbf{l}_{2}\right) F_{X X}\left(\mathbf{l}_{1}{ }^{\prime}, \mathbf{l}_{2}{ }^{\prime}\right) \\
& \times\left\{C_{\left|\mathbf{l}_{1}-\mathbf{l}_{1}{ }^{\prime}\right|}^{\phi \phi X} f_{X X}\left(-\mathbf{l}_{1}, \mathbf{l}_{1}{ }^{\prime}\right) f_{X X}\left(-\mathbf{l}_{2}, \mathbf{l}_{2}{ }^{\prime}\right)+C_{\left|\mathbf{l}_{1}-\mathbf{l}^{\prime}\right|}^{\phi \phi} f_{X X}\left(-\mathbf{l}_{1}, \mathbf{l}_{2}{ }^{\prime}\right) f_{X X}\left(-\mathbf{l}_{2}, \mathbf{l}_{1}{ }^{\prime}\right)\right\} \\
& N_{X X^{\prime}, X X^{\prime}}^{(1)}(L)=\frac{A_{X X^{\prime}}^{2}(L)}{L^{2}} \int \frac{d^{2} \mathbf{l}_{1}}{(2 \pi)^{2}} \int \frac{d^{2} \mathbf{l}_{1}^{\prime}}{(2 \pi)^{2}} F_{X X^{\prime}}\left(\mathbf{l}_{1}, \mathbf{l}_{2}\right) F_{X X^{\prime}}\left(\mathbf{l}_{1}{ }^{\prime}, \mathbf{l}_{2}{ }^{\prime}\right) \\
& \times \frac{1}{4}\left\{C _ { | _ { 1 } - \mathbf { l } _ { 1 ^ { \prime } | } | } ^ { \phi \phi } \left[f_{X X}\left(-\mathbf{l}_{1}, \mathbf{l}_{1}{ }^{\prime}\right) f_{X^{\prime} X^{\prime}}\left(-\mathbf{l}_{2}, \mathbf{l}_{2}{ }^{\prime}\right)+f_{X X^{\prime}}\left(-\mathbf{l}_{1}, \mathbf{l}_{1}{ }^{\prime}\right) f_{X^{\prime} X}\left(-\mathbf{l}_{2}, \mathbf{l}_{2}{ }^{\prime}\right)\right.\right. \\
& \left.\quad+f_{X^{\prime} X}\left(-\mathbf{l}_{1}, \mathbf{l}_{1}{ }^{\prime}\right) f_{X X^{\prime}}\left(-\mathbf{l}_{2}, \mathbf{l}_{2}{ }^{\prime}\right)+f_{X^{\prime} X^{\prime}}\left(-\mathbf{l}_{1}, \mathbf{l}_{1}{ }^{\prime}\right) f_{X X}\left(-\mathbf{l}_{2}, \mathbf{l}_{2}{ }^{\prime}\right)\right] \\
& +C_{\left|\mathbf{l}_{1}-\mathbf{l}_{2^{\prime} \mid}\right|}^{\phi \phi}\left[f_{X X^{\prime}}\left(-\mathbf{l}_{1}, \mathbf{l}_{2}{ }^{\prime}\right) f_{X^{\prime} X}\left(-\mathbf{l}_{2}, \mathbf{l}_{1}{ }^{\prime}\right)+f_{X X}\left(-\mathbf{l}_{1}, \mathbf{l}_{2}{ }^{\prime}\right) f_{X^{\prime} X^{\prime}}\left(-\mathbf{l}_{2}, \mathbf{l}_{1}{ }^{\prime}\right)\right. \\
& \left.\left.+f_{X^{\prime} X^{\prime}}\left(-\mathbf{l}_{1}, \mathbf{l}_{2}{ }^{\prime}\right) f_{X X}\left(-\mathbf{l}_{2}, \mathbf{l}_{1}{ }^{\prime}\right)+f_{X^{\prime} X}\left(-\mathbf{l}_{1}, \mathbf{l}_{2}{ }^{\prime}\right) f_{X X^{\prime}}\left(-\mathbf{l}_{2}, \mathbf{l}_{1}{ }^{\prime}\right)\right]\right\}
\end{aligned}
$$

The filters given in Eq. (A8) are no longer optimal in the presence of this additional noise, but the difference between these filters and the optimal filters should be negligible provided that $N_{X X, X X}^{(1)}(L) \ll N_{X X, X X}^{(0)}(L), N_{X X^{\prime}, X X^{\prime}}^{(1)}(L) \ll$ $N_{X X^{\prime}, X X^{\prime}}^{(0)}(L)$. For the purposes of power-spectrum estimation, the terms $N_{X X, X X}^{(1)}(L)$ and $N_{X X^{\prime}, X X^{\prime}}^{(1)}(L)$ are not only an additional contribution to the variance, but are also a systematic bias if not subtracted iteratively following Eq. (29).

A final point to consider is that the six different estimators $\mathbf{d}_{X X^{\prime}}(\mathbf{L})$ defined in this paper are not independent, as they are constructed from only three distinct maps. The covariance matrix for the six estimators will therefore not be diagonal, and this needs to be taken into account if the estimators are to be linearly combined to produce a single minimum-variance estimator. The off-diagonal elements of the covariance matrix can be evaluated in a straightforward manner involving pairs of double integrals similar to those of Eqs. (A9) and (A10).

[1] P. J. E. Peebles and J. T. Yu, Astrophys. J. 162, 815 (1970); R. A. Sunyaev and Ya. B. Zel'dovich, Astrophys. Space Sci. 7, 3 (1970); J. Silk, Astrophys. J. 151, 459 (1968); W. Hu and S. Dodelson, Ann. Rev. Astron. Astrophys. 40, 171 (2002).

[2] G. Jungman et al., Phys. Rev. D 54, 1332 (1995); J. R. Bond, G. Efstathiou, and M. Tegmark, Mon. Not. R. Astron. Soc. 291, L33 (1997); M. Zaldarriaga and D. N. Spergel, Astrophys. J. 488, 1 (1997); D. J. Eisenstein, W. Hu, and M. Tegmark, Astrophys. J. 518, 2 (1999).

[3] U. Seljak and M. Zaldarriaga, Phys. Rev. Lett. 82, 2636 (1999); M. Zaldarriaga and U. Seljak, Phys. Rev. D 58, 023003 (1998).

[4] M. Kesden, A. Cooray, and M. Kamionkowski, Phys. Rev. Lett. 89, 011304 (2002); L. Knox and Y.-S. Song, Phys. Rev. Lett. 89, 011303 (2002).

[5] W. Hu, Astrophys. J. 557, 79 (2001).

[6] W. Hu and T. Okamoto, Astrophys. J. 574, 2 (2001).

[7] F. Bernardeau, Astron. Astrophys. 324, 15 (1997); M. Zaldarriaga, Phys. Rev. D 62063510 (2000).

[8] W. Hu, Phys. Rev. D 62, 043007 (2000). 
[9] D. N. Spergel and D. M. Goldberg, Phys. Rev. D 59, 103001 (1999); D. M. Goldberg and D. N. Spergel, Phys. Rev. D 59, 103002 (1999); A. Cooray and W. Hu, Astrophys. J. 534, 533 (2000); M. Zaldarriaga and U. Seljak, Phys. Rev. D 59, 123507 (1999); H. V. Peiris and D. N. Spergel, Astrophys. J. 540, 605 (2000).

[10] R. A. Sunyaev and Ya. B. Zel'dovich, Mon. Not. R. Astron. Soc. 190, 413 (1980).

[11] A. Cooray, W. Hu, and M. Tegmark, Astrophys. J. 540, 1 (2000).

[12] L. Knox, Phys. Rev. D 52, 4307 (1995).

[13] M. Kesden, A. Cooray, and M. Kamionkowski, Phys. Rev. D 66, 083007 (2002).

[14] W. Hu, Astrophys. J. 529, 12 (2000).

[15] G. Giardino et al., Astron. Astrophys. 387, 82 (2002); F. R. Bouchet, S. Prunet, and S. K. Sethi, Mon. Not. R. Astron. Soc. 302, 663 (1999); S. Prunet, S. K. Sethi, and F. R. Bouchet, Mon. Not. R. Astron. Soc. 314, 348 (2000).

[16] M. Kamionkowski, A. Kosowsky, and A. Stebbins, Phys. Rev. Lett. 78, 2058 (1997); U. Seljak and M. Zaldarriaga, Phys. Rev. Lett. 78, 2054 (1997). 